\title{
The microscopic origin of the anomalous isotopic properties of ice relies on the strong quantum anharmonic regime of atomic vibration
}

Cite as: J. Chem. Phys. 155, 184502 (2021); https://doi.org/10.1063/5.0062689

Submitted: 07 July 2021 • Accepted: 20 October 2021 • Accepted Manuscript Online: 21 October 2021 • Published Online: 10 November 2021

Marco Cherubini, (D) Lorenzo Monacelli and Francesco Mauri
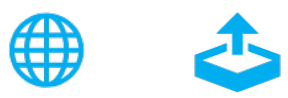

\section{ARTICLES YOU MAY BE INTERESTED IN}

Activity coefficients of aqueous electrolytes from implicit-water molecular dynamics simulations

The Journal of Chemical Physics 155, 184501 (2021); https://doi.org/10.1063/5.0064963

Local stress tensor calculation by the method-of-plane in microscopic systems with macroscopic flow: A formulation based on the velocity distribution function

The Journal of Chemical Physics 155, 184103 (2021); https://doi.org/10.1063/5.0062889

Ethane and methane at high pressures: Structure and stability

The Journal of Chemical Physics 155, 184503 (2021); https://doi.org/10.1063/5.0067828

\section{Chemical Physics Reviews}

\section{First Articles Now Onlinel = READ NOW »»}




\title{
The microscopic origin of the anomalous isotopic properties of ice relies on the strong quantum anharmonic regime of atomic vibration
}

\author{
Cite as: J. Chem. Phys. 155, 184502 (2021); doi: 10.1063/5.0062689 \\ Submitted: 7 July 2021 • Accepted: 20 October 2021 • \\ Published Online: 10 November 2021
}

Marco Cherubini, ${ }^{1,2, a)}$ (D) Lorenzo Monacelli, ${ }^{1, b)}$ (D) and Francesco Mauri ${ }^{1, c)}$

\begin{abstract}
AFFILIATIONS
1 Dipartimento di Fisica, Università di Roma Sapienza, Piazzale Aldo Moro 5, I-00185 Roma, Italy

${ }^{2}$ Center for Life NanoScience, Istituto Italiano di Tecnologia, viale Regina Elena 291, 00161 Rome, Italy
\end{abstract}

\author{
a) Author to whom correspondence should be addressed: marco.cherubini@uniroma7.it \\ b)Electronic mail: lorenzo.monacelli@roma7.infn.it \\ ${ }^{c}$ Electronic mail: francesco.mauri@uniroma7.it
}

\begin{abstract}
Water ice is a unique material presenting intriguing physical properties, such as negative thermal expansion and anomalous volume isotope effect (VIE). They arise from the interplay between weak hydrogen bonds and nuclear quantum fluctuations, making theoretical calculations challenging. Here, we employ the stochastic self-consistent harmonic approximation to investigate how thermal and quantum fluctuations affect the physical properties of ice XI with ab initio accuracy. Regarding the anomalous VIE, our work reveals that quantum effects on hydrogen are so strong to be in a nonlinear regime: When progressively increasing the mass of hydrogen from protium to infinity (classical limit), the volume first expands and then contracts, with a maximum slightly above the mass of tritium. We observe an anharmonic renormalization of about $10 \%$ in the bending and stretching phonon frequencies probed in IR and Raman experiments. For the first time, we report an accurate comparison of the low-energy phonon dispersion with the experimental data, possible only thanks to high-level accuracy in the electronic correlation and nuclear quantum and thermal fluctuations, paving the way for the study of thermal transport in ice from first-principles and the simulation of ice under pressure.
\end{abstract}

Published under an exclusive license by AIP Publishing. https://doi.org/10.1063/5.0062689

\section{INTRODUCTION}

Water is essential for life. It is ubiquitous on Earth in all states of aggregation and influences the climate, ${ }^{1}$ refrigeration, and transportation system. Ice is a molecular crystal composed of individual water molecules held to one another by hydrogen bonds, whose importance ranges from biology to astrophysics. Ice exhibits the polymorphism typical of molecular crystals. It has been found so far in 17 different crystalline structure phases, embodying the most complex phase diagram known in the literature. ${ }^{2-10}$ In each phase, the oxygen atoms are long-range ordered in a specific symmetry with the hydrogen atoms arranged around the oxygen according to the Bernal-Fowler ice rules. ${ }^{11}$ Of the 17 ice phases, some are proton-disordered, while the others are proton-ordered. In this work, we focus on the XI phase, ${ }^{3}$ that is, the proton-ordered counterpart of the ordinary ice $\mathrm{I}_{\mathrm{h}},{ }^{2}$ which is stable below $\mathrm{T}=72 \mathrm{~K}$ and was discovered by calorimetric measurements on $\mathrm{KOH}$ doped ice $\mathrm{I}_{\mathrm{h}} \cdot{ }^{12-14}$ This makes ice XI a prototype system for phase $\mathrm{I}_{\mathrm{h}}$. Ice XI has an orthorombic structure with space group $C m c 2_{1}$.

Liquid and crystalline water have been extensively studied in the last decades. Besides its pivotal role in biological processes, researchers have focused on the investigation of water because of its anomalous properties that attract scientific attention and make theoretical predictions challenging. The great difference in strength between the intermolecular hydrogen bonds and the intra-molecular covalent $\mathrm{OH}$ bonds produces a vibrational spectrum with a wide energy range, from low-energy rotons to high energy vibrons. ${ }^{15-17}$ The stretching vibrational modes of the water molecule have an energy of $3400 \mathrm{~cm}^{-1}$, which requires a temperature of $4900 \mathrm{~K}$ to populate the first excited state. Thus, the nuclear motion at room temperature is completely quantum mechanical. Anharmonic effects 
play a key role in ice, determining, for example, its anomalous thermal expansion ${ }^{18-20}$ at low temperatures, the inverse volume isotope effect (VIE) ${ }^{21-23}$ and the shifts in the vibrational spectra. The thermodynamic properties of ice have been investigated by using several approximations. Force-field and first-principles based path integral molecular dynamics (PIMD) and quasi-harmonic approximation (QHA) ${ }^{21-26}$ were employed to describe the negative thermal expansion and VIE, enlightening the strong dependence of the results on the force field or the density functional theory (DFT) functional used as well as some discrepancies of the QHA at high temperatures and the improvement of the simulations of water and ice obtained by including long-range van der Waals interactions. ${ }^{27-30}$ The vibrational properties of ice have been widely investigated by using mainly Raman, Infrared, and neutron spectroscopy. ${ }^{31-45}$ Alongside experimental works, several theoretical studies focused on the librational modes ${ }^{4-49}$ and on the $\mathrm{OH}$ stretching bands, ${ }^{50,51}$ where the authors were able to calculate linewidths in good agreement with experimental data but shifted peak positions. The low-energy modes computed using standard $a b$ initio techniques display a severe disagreement with experiments. This prevents the study of thermal transport properties in ice $a b$ initio and its characterization under pressure, an area of extensive experimental research. ${ }^{52-58}$ An accurate description of atomic vibrations is of paramount importance to reproduce thermodynamic and dynamical properties. In this work, we overcome the intrinsic limitations of other methodologies by using the selfconsistent harmonic approximation (SSCHA), ${ }^{59-62,82}$ which exploits a full-quantum variational principle on the free energy to account for the effect of anharmonicity arising from thermal and quantum fluctuations.

In Sec. II, we revise the methodology we employed in the simulations. In Sec. III A, we report the temperature evolution of volume (thermal expansion), internal geometry, and bulk modulus. We analyze the volume as a function of isotope mass in Sec. III B. Sections III C-III E deal with phonon dispersion, overtones and combination modes in the phonon spectrum, and the infrared and Raman vibrational spectrum of ice, respectively. In Sec. IV, we summarize the results and draw the conclusions.

\section{METHODS}

We work within the Born-Oppenheimer (BO) approximation $^{63}$ to separate electronic and nuclear degrees of freedom. The total electronic energy at fixed nuclei is calculated using a Neural Network Potential (NNP) developed in Ref. 64, trained on the revPBE0 ${ }^{65-67}$ functional with Grimme D3 dispersion correction $^{67,68}$ to properly account for long-range van der Waals interactions.

We solve the nuclear BO Hamiltonian using two different approximations: the quasi-harmonic approximation (QHA) and the self-consistent harmonic approximation (SSCHA).

In the QHA, the BO energy surface is expanded as a quadratic function around its minimum at each volume. The free energy is the sum of the BO energy $V\left(\mathcal{R},\left\{\vec{a}_{i}\right\}\right)$ at fixed nuclear position $\mathcal{R}$ and cell parameters $\left\{\vec{a}_{i}\right\}$, and the harmonic vibrational contribution,

$$
\mathcal{F}_{Q H A}\left(\mathcal{R},\left\{\vec{a}_{i}\right\}\right)=V\left(\mathcal{R},\left\{\vec{a}_{i}\right\}\right)+F_{v i b}\left(\mathcal{R},\left\{\vec{a}_{i}\right\}\right),
$$

where

$$
\begin{aligned}
F_{v i b}\left(\mathcal{R},\left\{\vec{a}_{i}\right\}\right)= & \frac{1}{N_{q}} \sum_{\boldsymbol{q} \in B Z} \sum_{\mu=1}^{3 N}\left[\frac{\hbar \omega_{q \mu}^{\mathcal{H}}\left(\mathcal{R},\left\{\vec{a}_{i}\right\}\right)}{2}\right. \\
& \left.+\frac{1}{\beta} \ln \left(1-e^{-\beta \hbar \omega_{q \mu}^{\mathcal{H}}\left(\mathcal{R},\left\{\vec{a}_{i}\right\}\right)}\right)\right] .
\end{aligned}
$$

Here, $N_{\boldsymbol{q}}$ is the number of $\boldsymbol{q}$ points in the Brillouin zone, $\beta=\left(k_{B} T\right)^{-1}$, and $\omega_{q \mu}^{\mathcal{H}}$ are the volume dependent harmonic frequencies in the $\boldsymbol{q}$ point for the $\mu$ mode. However, as in the harmonic model, the frequencies show no temperature dependence for a fixed volume.

The vector $\mathcal{R}$ describes the positions of the $N$ nuclei in the periodic cell (or supercell), while $\left\{\vec{a}_{i}\right\}$ are the unit cell vectors.

In principle, the QHA free energy is obtained by minimizing the functional $\mathcal{F}_{\mathrm{QHA}}$ in Eq. (1) at fixed volume and temperature. However, this minimization is computationally expensive for systems with many degrees of freedom like ice since it requires the calculation of the harmonic phonon frequencies for each value of the nuclear positions.

In this work (as commonly done), the QHA free energy is computed in the minimum $\boldsymbol{\mathcal { R }}_{0}$ of the BO energy $V\left(\mathcal{R},\left\{\vec{a}_{i}\right\}\right)$, obtained by relaxing both the internal coordinates and the cell vectors without vibrations at fixed pressure.

We overcome the intrinsic limitations of the QHA by employing a more sophisticated technique.

The self-consistent harmonic approximation (SSCHA) is a quantum variational principle on the free energy, which accounts for quantum and anharmonic effects on nuclei in a non-perturbative way. Within the SSCHA, we optimize the quantum density matrix to minimize the free energy, constraining the density matrix $\tilde{\rho}$ among Gaussians, uniquely defined by the average atomic positions (centroids) $\mathcal{R}$ and the quantum fluctuations around them (force constant matrix) $\boldsymbol{\Phi}$, just like any Gaussian is defined by the average and mean square displacements.

The SSCHA free energy is

$$
\mathcal{F}_{S S C H A}[\mathcal{R}, \Phi]=\left\langle V-\mathcal{V}^{\hat{\mathcal{H}_{\mathcal{R}}, \Phi}}\right\rangle_{\hat{\rho} \mathcal{R}, \Phi}+F_{v i b}(\mathcal{R}, \Phi),
$$

where $\mathcal{V}^{\hat{\mathcal{H}}, \Phi}$ is the potential energy for a trial harmonic Hamiltonian $\hat{\mathcal{H}}_{\mathcal{R}, \Phi}$ and the vibrational term has the same functional dependence as in Eq. (2). The average is computed in an ensemble of configurations generated according to the density matrix $\tilde{\rho}$.

The time-dependent extension of the SSCHA (TD-SSCHA) ${ }^{60,62}$ gives the possibility to compute the dynamical properties (phonon spectra).

The differences between the two approaches rely on three main points:

(a) The centroid position $\mathcal{R}$ : As mentioned earlier, we cannot optimize the centroids within the QHA at a reasonable computational cost, so we employ their equilibrium value without vibrations. In contrast, the minimization procedure in the SSCHA allows us to fully optimize the geometry at any temperature, including the average nuclear position $\mathcal{R}$.

(b) The frequencies: In the QHA approach, they are the harmonic frequencies. In the SSCHA framework, the frequencies 
are the eigenvalues of the dynamical matrix (the force constant matrix divided by the square root of the masses), obtained through the free energy minimization. Thus, they account for anharmonic quantum and thermal fluctuations.

(c) The SSCHA explicitly accounts for deviation of the real ionic energy landscape from the Harmonic approximation: $\left\langle V-\mathcal{V}^{\hat{\mathcal{H}}, \Phi}\right\rangle_{\rho_{\mathcal{R}, \Phi}}$ in Eq. (3). The inclusion of this term makes the SSCHA free energy variational with respect to the exact one. This property is not shared by the QHA free energy.

Both the harmonic calculation and the SSCHA calculation are performed in supercells with periodic boundary conditions.

In Appendix D, we report a detailed discussion about the convergence properties in the SSCHA and QHA. We found that the results are converged for a $3 \times 3 \times 2$ supercell in the SSCHA and a $14 \times 14 \times 14$ supercell for the QHA.

Although an approximate technique, this self-consistent approach revealed to perform quite accurately in describing free energy differences (see Ref. 69) that determine the properties investigated in this paper. Moreover, the large amount of simulations performed in our work prevents the use of more accurate path integral methods. In addition, most of the properties, such as the VIE, are studied at zero temperature, a limit that is computationally very expensive and difficult to achieve through PI simulations. Furthermore, PI is a static theory, and it requires alternative formulations to describe dynamical spectral functions, while the TD-SSCHA is a dynamical theory that grants direct access to the spectral functions.

For each temperature, we estimated the equilibrium volume $\Omega_{e q}(T)$ as the one where the pressure, defined as the derivative of the free energy with respect to a strain tensor $\varepsilon, P=-1 / \Omega \partial \mathcal{F} / \partial \boldsymbol{\varepsilon}$, vanishes,

$$
\Omega_{e q}(T): P\left(\Omega_{e q}(T), T\right)=0 .
$$

In the SSCHA framework, we have an analytical equation to compute the pressure for each simulation, ${ }^{61}$ while we employed the finite difference approach for the QHA.

When an external pressure is applied to a solid, its volume changes relatively; the bulk modulus is how a crystal withstands modifications of volume under pressure,

$$
B(T)=-\left.\Omega_{e q}(T) \frac{\partial P(\Omega, T)}{\partial \Omega}\right|_{\Omega_{e q}(T)} .
$$

\section{RESULTS}

We report a detailed investigation of the phase XI of ice, the proton-ordered phase of common ice, ${ }^{12}$ stable below $72 \mathrm{~K}$. Quantum anharmonic effects on the nuclei affect the properties of the hydrogen bonds, producing exotic behaviors, such as negative thermal expansion or anomalous VIE. Soft intermolecular hydrogen bonds coexist with harder intra-molecular covalent $\mathrm{OH}$ bonds producing phonons with a very wide energy range, heavily impacted by anharmonicity.

It is known in the literature that the commonly used QHA produces accurate predictions of thermodynamic properties at low temperatures ( $T \leq 100 \mathrm{~K})$, manifesting instead some inaccuracies at higher temperatures. Therefore, we are using both the QHA and the SSCHA, a more sophisticated technique to account for quantum fluctuations, to simulate the system, and to compare their outcomes.

Section III A is dedicated to the anomalous thermal expansion of ice, the bulk modulus, and the temperature dependence of the crystalline properties.

\section{A. Thermodynamic properties}

The absolute value of the equilibrium volume per $\mathrm{H}_{2} \mathrm{O}$ molecule is in Fig. 1(a). We notice a considerable effect of the
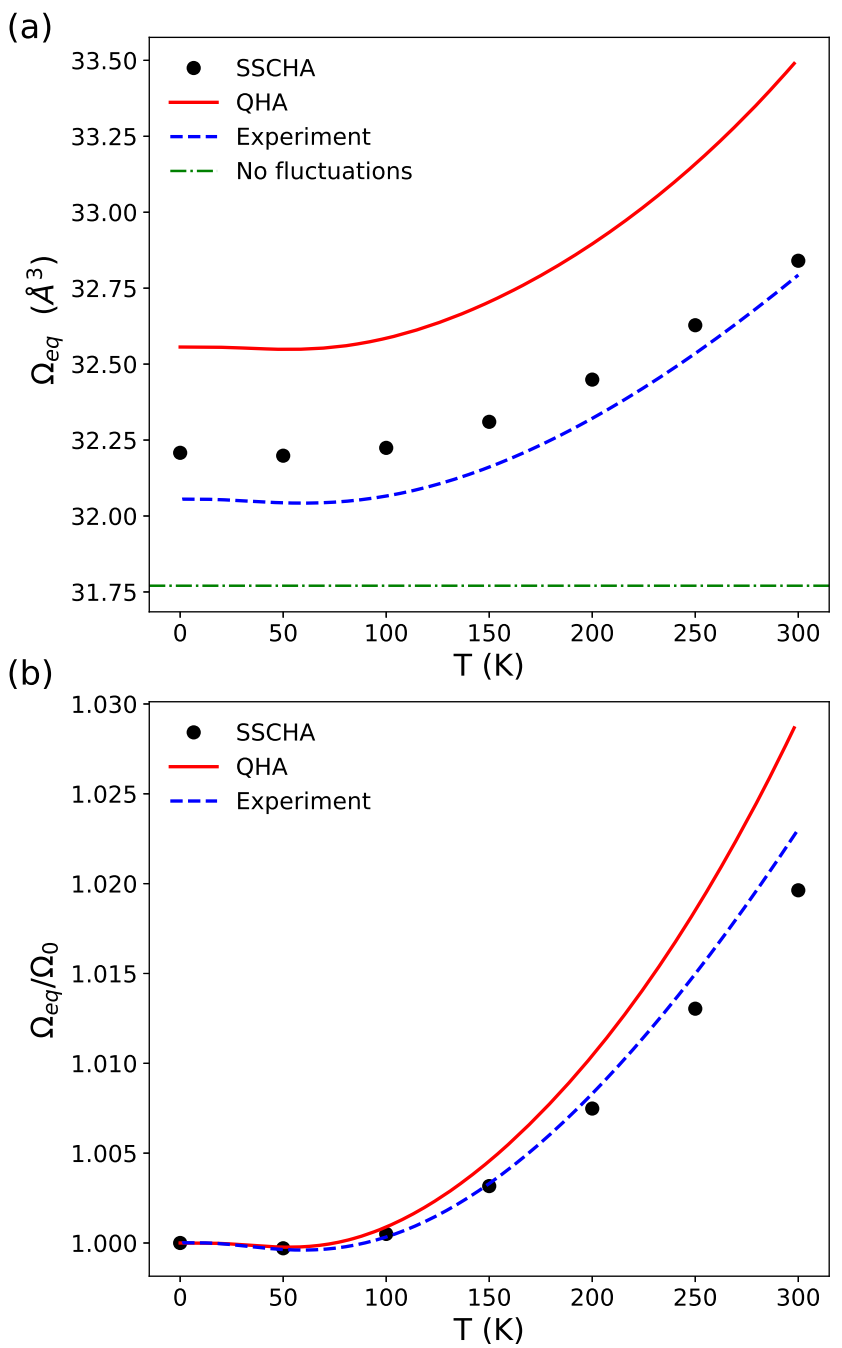

FIG. 1. Equilibrium volume per $\mathrm{H}_{2} \mathrm{O}$ molecule of ice as a function of temperature. (a) Comparison of the absolute value of the volume in the SSCHA (black circles) and QHA (solid red line) with the experimental measurements in Ref. 19 (blue dotted line). The classical equilibrium volume, where thermal and quantum fluctuations are neglected, is the green dotted-dashed line. (b) The normalized equilibrium volume $\Omega_{e q}(T) / \Omega_{e q}(T=0)$ in the SSCHA (black circles) and QHA (red solid line) is compared to the experiment (blue dotted line). 
zero point motion that shifts the curves one respect to the other. The zero-temperature equilibrium volumes predicted by the different theories and their percentage shift with respect to the classical limit are reported in the first part of Table I. The agreement with experiment is much better in the SSCHA than in the QHA picture. These data are discussed in detail in Sec. III B, concerning the isotope volume effect. Since $\Omega_{e q}(T=0)$ is theory dependent, to compare the thermal expansion, in Fig. 1(b), we report the normalized volume $\Omega_{e q}(T) / \Omega_{e q}(T=0)$.

In the low-temperature regime, $T \leq 50 \mathrm{~K}$, the predictions for the normalized equilibrium volume in the QHA and SSCHA are very similar and match experiment ${ }^{19}$ closely. For higher temperatures, essential differences between the two theories arise. The SSCHA agrees with experimental measurements within a $0.1 \%$ up to $200 \mathrm{~K}$. The QHA, otherwise, deviates from the experimental data above $100 \mathrm{~K}$. This establishes that SSCHA theory is able to accurately describe the thermal expansion of ice, overcoming the limitations of commonly used techniques based on the harmonic approximation.

Equation (5) suggests a direct dependence between equilibrium volume and bulk modulus. The absolute value and the normalized one $B(T) / B(T=0)$ of the bulk modulus are reported in Fig. 2. Already at $T=0 \mathrm{~K}$, the bulk modulus is strongly renormalized by ionic quantum fluctuations by $22 \%$ and $15 \%$ in the QHA and SSCHA, respectively [Fig. 2(a)].

The bulk modulus has an anomalous strong temperature dependence; experimental data ${ }^{19}$ show a $20 \%$ reduction from 0 to $300 \mathrm{~K}$. The SSCHA reproduces this behavior excellently [Fig. 2(b)], while the QHA overestimates the bulk modulus reduction by $10 \%$. This strong temperature dependence originates by $64 \%$ from volume expansion and the remaining $36 \%$ from vibrational free energy. We refer to Appendix $\mathrm{E}$ for further details.

This result is fundamental to geophysics, where the compressibility of a solid is of paramount importance for studying the inner composition of Earth. ${ }^{70,71}$ The combined employment of high accuracy in the electronic exchange-correlation and the accurate description of quantum nuclear motion provided by the SSCHA correctly describe the thermodynamics properties of ice beyond the accuracy of quasi-harmonic simulations.

TABLE I. The first part reports the equilibrium volume per water molecule of ice at $\mathrm{T}=0 \mathrm{~K}$. In the first row, there is the comparison of the volume computed in the QHA and SSCHA with the experiment ${ }^{19}$ and with the classical result, where quantum and thermal fluctuations are neglected. In the second row, we report the percentage shift with respect to the classical volume. The second part shows the equilibrium volume per water molecule in the QHA when free energy of Eq. (1) is computed using the SSCHA equilibrium positions $\{\boldsymbol{\mathcal { R }}\}_{\text {SSCHA }}$ or using both the SSCHA positions and frequencies and their error with respect to the SSCHA result.

\begin{tabular}{lcccc}
\hline \hline & Classic & QHA & SSCHA & Expt. ${ }^{19}$ \\
\hline$\Omega_{\text {eq }}\left(\AA^{3}\right)$ & 31.771 & 32.555 & 32.207 & 32.055 \\
$\%$ & 0 & 2.47 & 1.38 & \\
\hline & QHA @ $\{\mathcal{R}\}_{\text {SSCHA }}$ & QHA @ $\{\mathcal{R}, \omega\}$ ssCHA \\
\hline$\Omega_{\text {eq }}\left(\AA^{3}\right)$ & 32.613 & & 32.068 \\
$\Delta\left(\AA^{3}\right)$ & 32.068 & -0.139 \\
\hline \hline
\end{tabular}

(a)
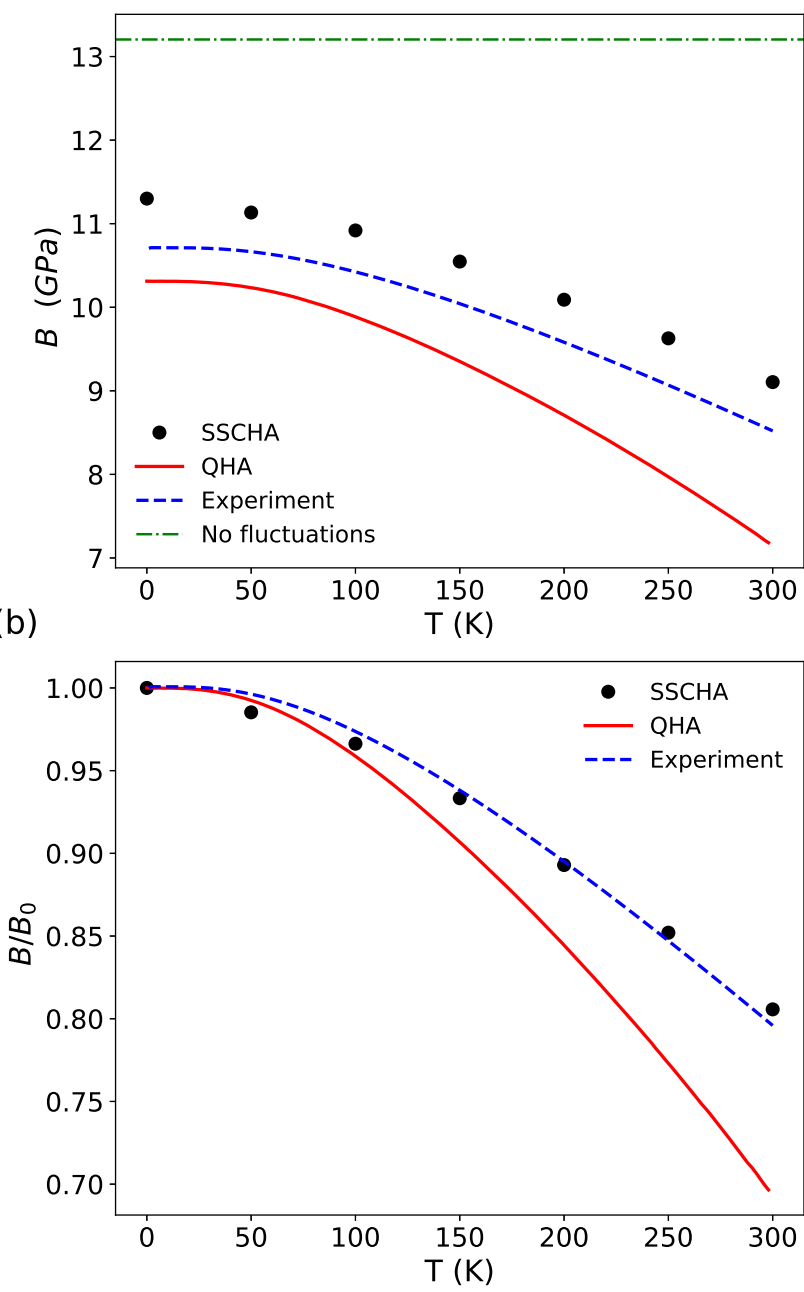

FIG. 2. (a) Temperature dependence of the bulk modulus. The QHA (red solid line) and SSCHA (black circles) results are compared with the experiment ${ }^{19}$ (blue dashed line). The green dotted-dashed line is the classical value for the bulk modulus, obtained by neglecting thermal and quantum fluctuations. (b) Comparison of the normalized bulk modulus $B(T) / B(T=0)$ in QHA and SSCHA with the experiment. The bulk modulus is computed as in Eq. (5).

The SSCHA also provides information about the geometry of ice. We report the temperature dependence of the average covalent bond length $\mathrm{OH}$ in Fig. 3. We refer to Appendix E for the description of the hydrogen bond.

Counterintuitively, the water molecules shrink upon heating at high temperatures (Fig. 3). This is only marginally a consequence of intermolecular hydrogen bond weakening due to the increasing distance between molecules with temperature, but rather a complex effect of anharmonicity triggered by molecular vibration. If we relax the structure with static nuclei at the SSCHA equilibrium volume for each temperature (Fig. 3, red squares), we explain only $15 \%$ of this effect. 


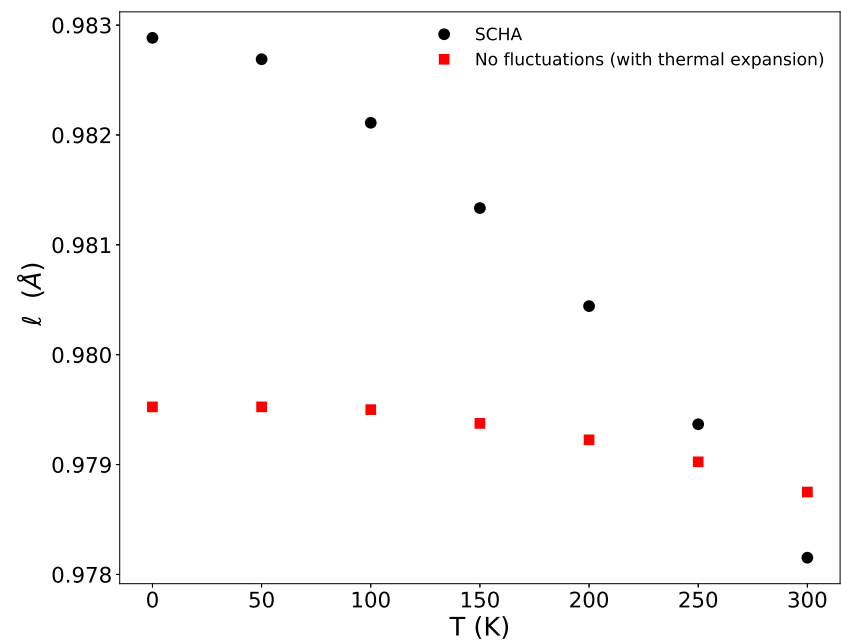

FIG. 3. Temperature dependence of the covalent bond length. The SSCHA bond length (black circles) is compared with the classical result (red squares), computed from the minimization of the BO energy $V\left(\mathcal{R},\left\{\vec{a}_{i}\right\}\right)$, where the effect of the thermal expansion is introduced by fixing the volume to be the SSCHA equilibrium one at each temperature, in order to point out the contribution of thermal and quantum fluctuations.

Since the vibration that deforms the water molecule is a stretching mode, with $\omega \simeq 3400 \mathrm{~cm}^{-1}$ and excitation temperature $T \simeq 4900 \mathrm{~K}$, this large temperature dependence can only be explained by the anharmonic interaction between translational molecular modes, the only ones whose population changes in this temperature range, and the stretching mode that affects the $\mathrm{OH}$ bond length.

\section{B. Volume isotope effect}

According to classical mechanics, the equilibrium volume does not depend on the mass of the atoms, and, thus, it is isotope independent. Quantum effects overturn this simple situation. In most crystalline systems, the heavier the isotopes, the smaller the equilibrium volume. In rare exceptions, like ice, by substituting hydrogen with deuterium the equilibrium volume increases. ${ }^{21,22}$ This is known as anomalous volume isotope effect.

In Table I, we report the equilibrium volume per water molecule at zero temperature with protium mass of hydrogen, computed without thermal fluctuations, with quantum effects and harmonic phonons (QHA), and with full anharmonic quantum zero point motion (SSCHA) compared with the experiment in Ref. 19.

As seen, the quantum anharmonic theory is the closest match with the experiment, with an error smaller than $0.5 \%$. The discrepancy between the SSCHA results and those obtained within the QHA, which account for volume effects but not for vibrational anharmonicity, is comparable in size to neglecting ionic motion altogether.

Anharmonicity affects the results in two ways: It changes the average position of nuclei (see Fig. 3) and modifies the vibrational frequencies. To shed light on which effect dominates the volume expansion, we repeated the harmonic calculation by fixing nuclear positions to the SSCHA result (QHA @ $\{\boldsymbol{\mathcal { R }}\}_{\text {SSCHA }}$ ) and by employing also the frequencies shifted by the anharmonicity (QHA @ $\left.\{\boldsymbol{\mathcal { R }}, \omega\}_{\text {SSCHA }}\right)$. The results are reported in the second part of Table I. The last row indicates the volume difference with respect to the full anharmonic theory.

This analysis reveals the origin of the discrepancies between a quasi-harmonic approach and a full non-perturbative anharmonic treatment of nuclear vibrations, unveiling how the key role played by anharmonicity is on the frequency renormalization rather than the significative structural changes.

Having elucidated the crucial role of anharmonicity in correctly describing the volume expansion, we systematically explore the effect of varying the masses of both the hydrogen and the oxygen isotopes on the volume.

At first, we investigate the dependence of the equilibrium volume at $\mathrm{T}=0 \mathrm{~K}$ on the hydrogen mass; numerical simulations give the possibility to modify this parameter without limitations, while experiments are available only for hydrogen and deuterium. ${ }^{18,19}$

In Fig. 4, we compare the equilibrium volumes per water molecule obtained at different levels of the theory with two experimental measurements. ${ }^{18,19}$ The solid line is the classical limit used as a reference. The numerical values for the volume difference at $\mathrm{T}=0 \mathrm{~K}$ in the theoretical models and the experiments are reported in Table II. The anharmonic theory (SSCHA) correctly predicts the sign of the VIE, while the quasi-harmonic approach fails, predicting a volume reduction. However, the SSCHA heavily overestimates the experiment, resulting in a volume difference from 3.8 to 6.7 larger than the measured data.

Experimental data are measured on hydrogen-disordered samples of ice $\mathrm{I}_{\mathrm{h}}$, while the simulation is performed on the hydrogenordered ice XI. To unveil the role of hydrogen ordering, we repeated the calculation of the VIE in a hydrogen-disordered structure for ice

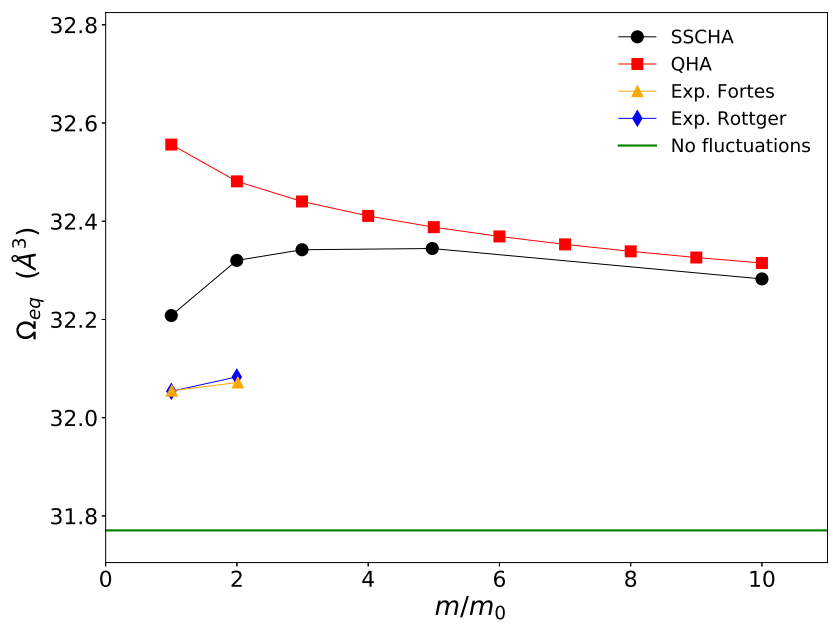

FIG. 4. Dependence of the equilibrium volume per water molecule of ice on the hydrogen mass at $\mathrm{T}=0 \mathrm{~K}$. The results obtained in the $\mathrm{QHA}$ (red squares) and in the SSCHA (black circles) are compared with two experimental results (yellow triangles and blue diamonds). ${ }^{18,19}$ The mass independent classical equilibrium volume is reported as a reference (green solid line). 
TABLE II. Equilibrium volume per water molecule difference $\Omega_{\mathrm{D}_{2} \mathrm{O}}-\Omega_{\mathrm{H}_{2} \mathrm{O}}$ at $\mathrm{T}=0 \mathrm{~K}$. The SSCHA and QHA results computed in the converged meshes are compared with two experimental measurements. ${ }^{18,19}$ The last column shows the volume difference computed in the SSCHA by using the NNP RPBE-D3 functional devised in Ref. 64 in order to analyze the dependence of the VIE on the functional used.

\begin{tabular}{cccccc}
\hline \hline & SSCHA & QHA & Expt. $^{19}$ & Expt. $^{18}$ & SSCHA RPBE-D3 \\
\hline$\Delta \Omega\left(\AA^{3}\right)$ & 0.112 & -0.075 & 0.017 & 0.029 & 0.156 \\
\hline \hline
\end{tabular}

$\mathrm{I}_{\mathrm{h}}$. We generated disordered cells containing 24 atoms, and we ran convergence tests (not shown here) using supercells containing up to 432 atoms ( 144 water molecules). We found converged properties for supercells with 192 atoms (64 molecules). This result is in agreement with previous theoretical works, ${ }^{64,72}$ where 64 molecules simulation cells are used to attain convergence.

We obtained a difference in volume, which deviates by $3.5 \%$ from that observed for the hydrogen-ordered structure, unveiling that hydrogen ordering does not significantly affect the VIE, and it is not the origin of the discrepancy between theory and experiments.

Our calculation's most relevant source of error is in the electronic correlation: We repeated the simulation employing a different electronic energy engine (a neural network trained on Revised Perdew-Burke-Ernzerhof (RPBE) with Grimme D3 dispersion correction $^{64}$ ). We obtained a difference in volume, deviating by $38 \%$ from the original simulation, giving a rough estimate of the error introduced by the DFT functional.

The QHA and SSCHA volumes are different for the physical isotopes of hydrogen (protium, deuterium, and tritium). The difference disappears as we increase the hydrogen mass. This derives from the reduced role of anharmonicity for higher mass where quantum fluctuations at zero temperature are smaller. The crossover above which the QHA correctly reproduces the VIE occurs for an isotope mass of five times the hydrogen's one, not a stable isotope. This means that the quantum regime of protium, deuterium, and tritium is anharmonic, beyond the range of validity of the quasi-harmonic theories.

In Fig. 5, we show the equilibrium volume of the solid when the mass of each atomic species (hydrogen and oxygen) is varied separately until the classical limit of infinite mass is reached, as well as the evolution of the equilibrium volume when the mass of the whole molecule is increased. Obviously, there are only few of these combinations in nature that are stable, but we can infer the quantum nature of each element from this plot.

By increasing the mass of the whole solid, we manage to approach the classical limit (the continuous line) for $m / m_{\mathrm{H}_{2} \mathrm{O}} \gtrsim 10000$.

The largest natural atomic species weighs only 238 times the mass of protium. This unveils how quantum effects on nuclei, usually neglected in atomistic calculations, are of paramount importance even with "heavy" atoms. This is further proved by the isotope volume effect of oxygen that, scaled to its much lower mass ratio between its natural isotopes, is bigger than hydrogen.

Figure 5 reveals a nonmonotonous volume expansion compared with quantum fluctuations for the hydrogen isotopes. First, the equilibrium volume expands when we increase the mass,

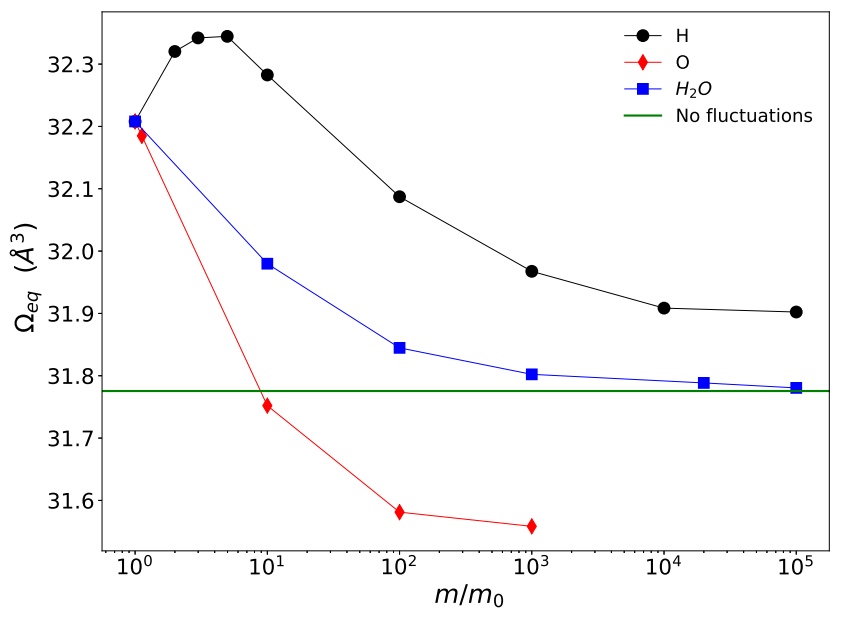

FIG. 5. Volume isotope effect for all the atomic species in ice XI in the SSCHA framework at $\mathrm{T}=0 \mathrm{~K}$. The black circles indicate the equilibrium volume per $\mathrm{H}_{2} \mathrm{O}$ molecule when the hydrogen mass is increased by keeping the oxygen mass fixed to its ${ }^{16} \mathrm{O}$ isotope value. The red diamonds show the equilibrium volume when the oxygen mass is varied with fixed hydrogen mass. The blue squares stand for the equilibrium volumes when the mass of the entire water molecule is increased until reaching the classical limit shown as a reference (green solid line).

reaching the maximum value for $m \simeq 5 m_{H}$; then, we observe a contraction to the classical value for bigger masses. This behavior explains that the VIE is due to a crucial nonlinear regime of quantum fluctuations in ice, overturning the hypothesis of a monotonous volume reduction due to quantum effects. This exotic behavior cannot be explained in a quasi-harmonic picture, as evident from Fig. 4.

\section{Phonon dispersion}

The coexistence of strong intra-molecular and weak intermolecular bonds in ice produces a vast vibrational spectrum. To compare with experimental results, we computed the real phonons from the dynamical interacting Green function within the timedependent SSCHA ${ }^{60,62,82}$ (TD-SSCHA) to account for dynamical quantum anharmonic effects (see Appendices $\mathrm{A}$ and $\mathrm{F}$ for further details). We employed the static approximation of the selfenergy for the low-energy modes, as described in Appendix A and Refs. 60 and 73.

We compare the harmonic phonons and the anharmonic (SSCHA) phonons for $\mathrm{D}_{2} \mathrm{O}$ ice at $\mathrm{T}=140 \mathrm{~K}$ and $\mathrm{P}=0.05 \mathrm{GPa}$ with the experiment ${ }^{55}$ in Fig. 6 . The details on the computation are given in Appendix F.

The harmonic acoustic phonons are in good agreement with the experiment and deviate significantly from experimental data at high frequency $\left(\omega \geq 120 \mathrm{~cm}^{-1}\right)$. Anharmonic effects correct the deviation, obtaining an excellent match between theory and experimental data. The harmonic energy of the lowest acoustic branch overestimates the speed of sound of $20 \%$, introducing a substantial error in the determination of thermal transport properties, further stressing the fundamental role of anharmonicity in thermal conductivity. 


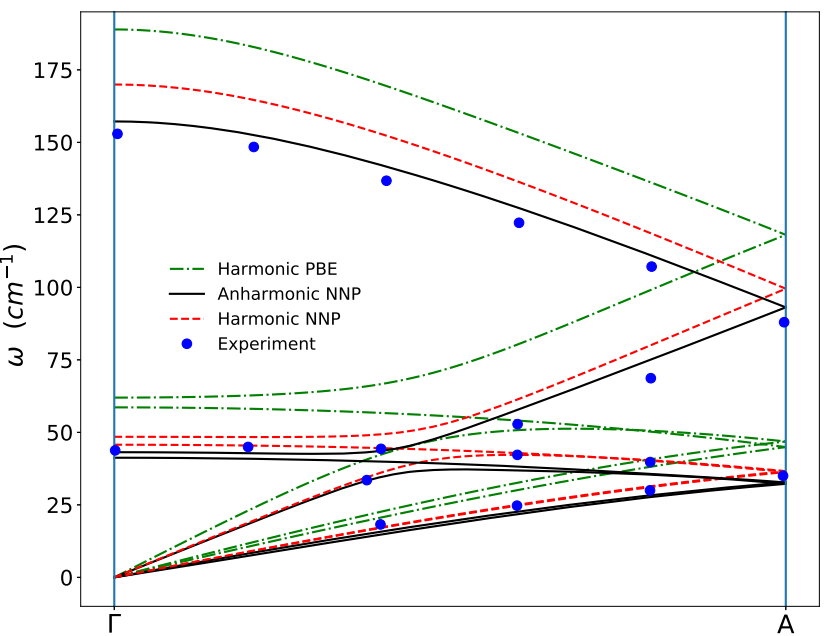

FIG. 6. Comparison of the low-energy phonon dispersion (molecular translations) with the experiment ${ }^{55}$ (blue circles) for deuterated ice at $\mathrm{T}=140 \mathrm{~K}$ and $P=0.05 \mathrm{GPa}$. Harmonic (red dashed lines) and anharmonic phonons (black solid line) computed as the poles of the interacting one-phonon Green function in the static limit (using the hybrid revPBE0-D3 functional fitted with the NNP ${ }^{64}$ ) are shown. The effect of the DFT functional on the harmonic phonon dispersion is reported by showing the harmonic dispersion calculated with the $\mathrm{PBE}^{74}$ functional (green dotted-dashed lines).

We report an excellent agreement between experimental data and a simulation of ice with $a b$ initio accuracy for the first time. This result has a profound impact, enabling the first-principles simulation of thermal transport, where an accurate description of the acoustic phonons is required. Moreover, low-energy phonons are the only modes detectable at high pressure; our work paves the way to characterize ice under pressure further.

The astonishingly good agreement we achieve is merit of the combined effect of the correct treatment of anharmonicity and of the electronic functional adopted for the calculation: The harmonic phonons with Perdew-Burke-Ernzerhof (PBE), the common choice in $a b$ initio atomistic simulations of water, ${ }^{22,75,76}$ are reported in Fig. 6 (green dotted-dashed lines), offering a comparison with phonons computed with NN-revPBE0 (employed in our work). We notice a considerable dependence on the DFT functional. The use of the NNP improves the PBE harmonic dispersion, where the error committed approximating the experimental points ranges from $30 \%$ to $36 \%$, e.g., in the A point.

\section{Spectral function}

The phonon spectral function $\sigma(q, \omega)$ gives access to the quasiparticles' energies and lifetime. The spectral function is proportional to the signal probed in scattering experiments, as neutron or x-ray scattering, and it is computed from the diagonal elements of the dynamical one-phonon Green function $G(q, \omega)$,

$$
\sigma(q, \omega)=-\frac{\omega}{\pi} \operatorname{Tr} \operatorname{Im} G(q, \omega)
$$

The details for the calculation are reported in Appendix B.
The phonon density of states (DOS) computed with the SSCHA dynamical matrix at equilibrium (without the self-energy correction) describes anharmonic non-interacting phonons, while the dynamical spectral function encapsulates all the effects of phonon-phonon interactions, where the addition of the self-energy term [Eq. (A7)] may produce a combination of modes [see Eqs. (A4) and (A5) in Appendix A].

In Fig. 7, we report the comparison between the phonon DOS and the spectral function at $\Gamma$ of $\mathrm{H}_{2} \mathrm{O}$ ice to enlighten the presence of combination modes and anharmonic overtones $\left(\mathrm{D}_{2} \mathrm{O}\right.$ is described in Appendix $G$ ). We reveal the presence of two structures in the spectral function that are absent in the DOS: One occurs at energies between the bending and stretching bands, the other occurs at twice the frequency of the stretching modes.

The origin of the observed combination modes is investigated in detail in Appendix G. Specifically, we find that the first combination mode originates from the interaction between the libration and bending bands, while the anharmonic overtone emerges from the interaction of the stretching modes with themselves.

From the spectral function, we can extract the real phonon energies and their lifetimes, as shown in Appendix A. We report in Table III the phonon energies and their linewidths for few selected intense modes at $\mathrm{T}=0 \mathrm{~K}$ and $\mathrm{T}=300 \mathrm{~K}$ in the Brillouin zone center.

We observe an essential reduction of the lifetime increasing the temperature. The phonon energies are less temperature-dependent than their lifetime. Stretching modes gain energy upon heating, while all the others become softer.

\section{E. Spectroscopy}

Anharmonicity shifts the frequencies of phonons and introduces a finite lifetime. Here, we simulate the Raman and IR

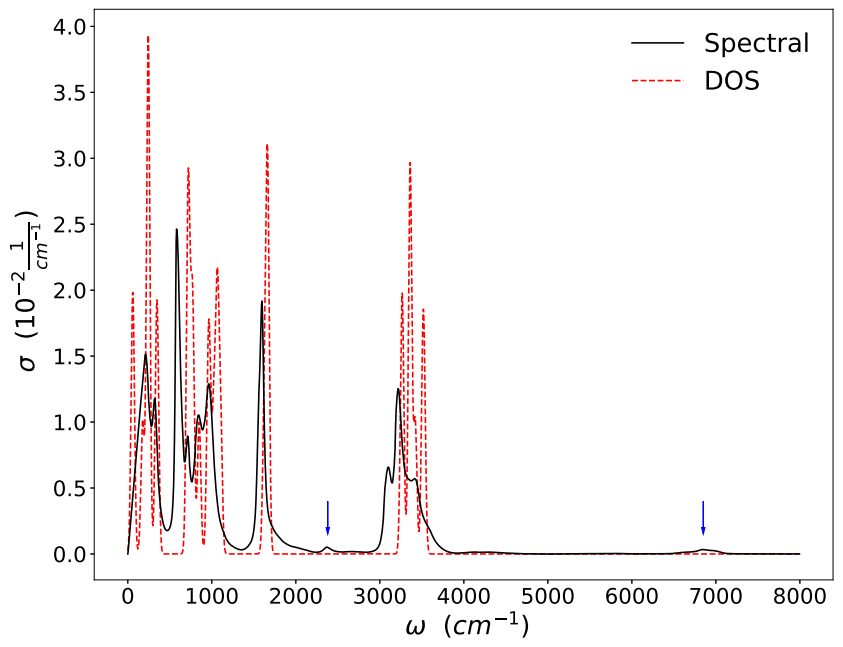

FIG. 7. Comparison of the density of states and spectral function at $\Gamma . \mathrm{H}_{2} \mathrm{O}$ ice at $\mathrm{T}=200 \mathrm{~K}$ and ambient pressure. The spectral function (black solid line) and DOS (red dashed line) are shown. The DOS is computed by using the SSCHA dynamical matrix at equilibrium without the inclusion of the self-energy term and by adding an artificial smearing factor of $20 \mathrm{~cm}^{-1}$. The blue arrows indicate the combination modes and anharmonic overtones. 
TABLE III. Peak frequency and linewidths of some selected intense modes in $\mathrm{H}_{2} \mathrm{O}$ ice at ambient pressure for two values of temperature, $T=0$ and $300 \mathrm{~K}$ at $\Gamma$. The first column indicates to which band the modes belong. See Appendix A for details about the calculations of frequencies and linewidths.

\begin{tabular}{lccccc}
\hline \hline & \multicolumn{2}{c}{$\mathrm{T}=0 \mathrm{~K}$} & & \multicolumn{2}{c}{$\mathrm{T}=300 \mathrm{~K}$} \\
\cline { 2 - 3 } \cline { 5 - 6 } & $\Omega_{\mu}\left(\mathrm{cm}^{-1}\right)$ & $\Gamma_{\mu}\left(\mathrm{cm}^{-1}\right)$ & & $\Omega_{\mu}\left(\mathrm{cm}^{-1}\right)$ & $\Gamma_{\mu}\left(\mathrm{cm}^{-1}\right)$ \\
\hline tr. & 208 & 12 & & 211 & 75 \\
& 326 & 11 & & 311 & 52 \\
& 602 & 4 & & 557 & 20 \\
lib. & 740 & 5 & & 682 & 43 \\
& 851 & 12 & & 808 & 47 \\
& 968 & 16 & & 940 & 54 \\
bend. & 1565 & 16 & & 1551 & 31 \\
& 1606 & 20 & & 1594 & 40 \\
str. & 3102 & 13 & & 3080 & 68 \\
& 3207 & 16 & 3237 & 77 \\
\hline \hline
\end{tabular}

vibrational spectroscopy on ice, adequately accounting for quantum and thermal anharmonic nuclear motion.

Raman scattering and infrared absorption are complementary tools to probe phonon energies at $\Gamma$. The former is based on an inelastic scattering process that detects modes due to changes in the polarizability, while the latter relies on the absorption process and the vibrations detected involve modifications of the dipole moment. Consequently, the selection rules for the two spectroscopies are different, and often active IR modes are Raman inactive (or vice versa).

Appendix C and Refs. 62 and 77 describe the relationships between the Raman and IR signals and the anharmonic phonon Green functions.

In Fig. 8(a), we compare the simulated Raman spectra with the experiment ${ }^{42}$ for the same geometry $c\left(a,{ }^{*}\right) b$ (see Appendix $C$ ) in $\mathrm{H}_{2} \mathrm{O}$ ice XI at $\mathrm{T}=65 \mathrm{~K}$. We report deuterated ice at $\mathrm{T}=269 \mathrm{~K}$ in the c(a, a)b geometry ${ }^{37}$ in Fig. 8(b).

The result obtained in the anharmonic dynamical theory (TD-SSCHA) matches excellently with the experimental results, correcting a shift of the harmonic phonon energy in the stretching modes of about $10 \%(7 \%)$ of the energy in $\mathrm{H}_{2} \mathrm{O}\left(\mathrm{D}_{2} \mathrm{O}\right)$ ice. The theory can predict the presence of the combination mode [indicated by the blue arrow in Fig. 8(a)] for $\mathrm{H}_{2} \mathrm{O}$ ice in the considered geometry. Instead, this mode has very low intensity in the experimental geometry of $\mathrm{D}_{2} \mathrm{O}$ ice.

Finite linewidths in the harmonic model are for presentation purposes only, as harmonic phonons have infinite lifetimes.

Phonon vibrations impact the low-energy optical properties of any material. In Fig. 9, we report the imaginary part of the dielectric function at $200 \mathrm{~K}$ of $\mathrm{H}_{2} \mathrm{O}$ ice. To include all the crystal orientations, we plot the trace of the dielectric tensor as in Eq. (C6). As for the Raman, anharmonicity reduces by $10 \%$ the harmonic stretching band energy, providing excellent agreement with the experiment. ${ }^{38}$ In addition, the combination mode located at $2300 \mathrm{~cm}^{-1}$ (highlighted by the blue arrow in Fig. 9) observed experimentally is correctly reproduced by the anharmonic spectrum.
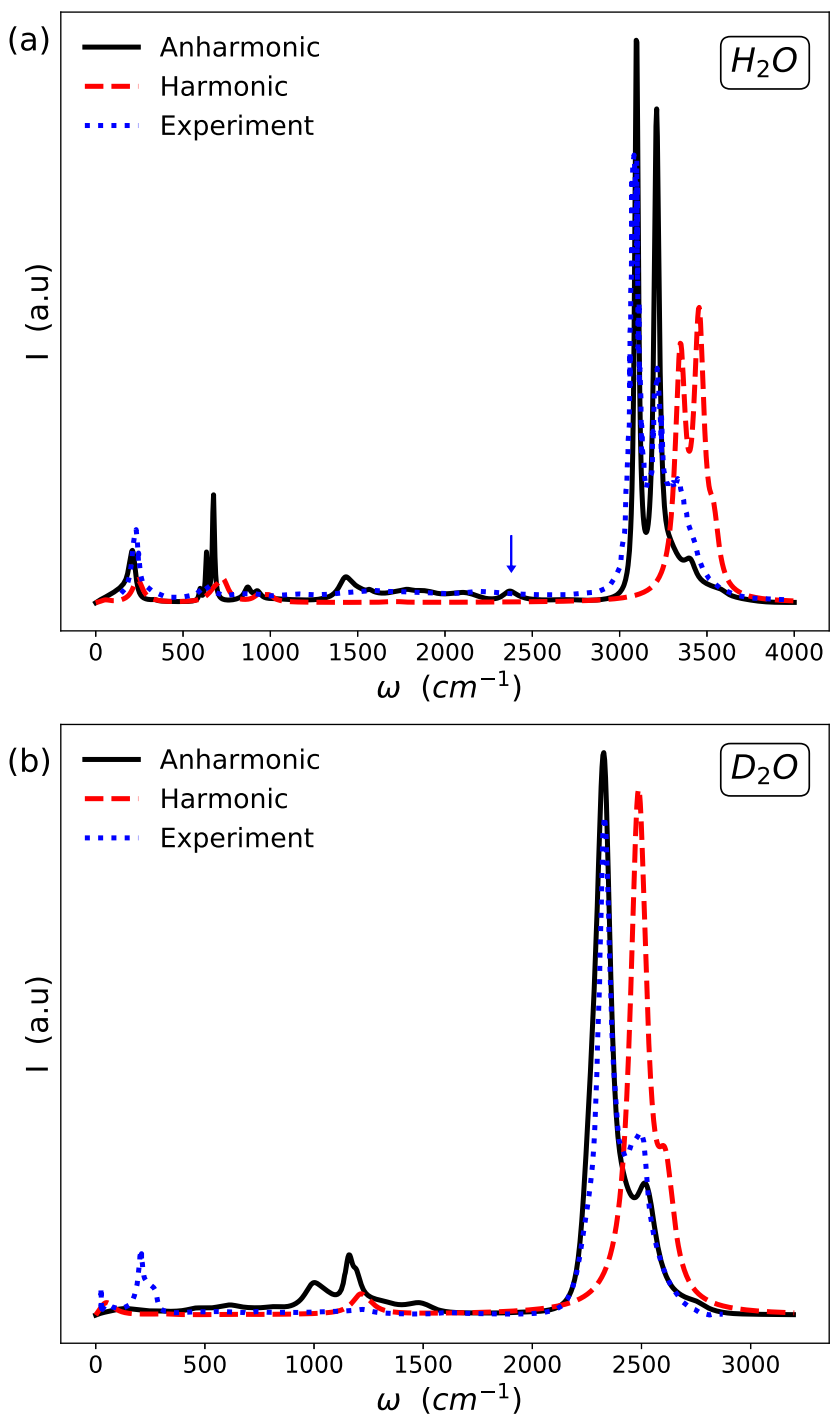

FIG. 8. (a) Raman scattering spectra at $\mathrm{T}=65 \mathrm{~K}$ in $\mathrm{H}_{2} \mathrm{O}$ ice $\mathrm{XI}$ in the $\mathrm{a}\left(\mathrm{c},{ }^{*}\right) \mathrm{b}$ geometry. The experimental spectrum ${ }^{42}$ (blue dotted line) is compared with the harmonic (red dashed line) and the anharmonic phonons (solid black line), computed in the SSCHA framework with the inclusion of the bubble term in Eq. (A7). (b) Raman scattering spectra for deuterated ice at $\mathrm{T}=269 \mathrm{~K}$ in the $\mathrm{c}(\mathrm{a}$, a)b geometry. Comparison between harmonic (red dashed line) and anharmonic SSCHA phonons with the bubble correction (solid black line) spectra, and the experiment ${ }^{3}$ (blue dotted line). Blue arrows indicate the position of the combination mode. An artificial broadening is employed in the harmonic approximation to guide the eyes in the comparison of the spectrum with experiment of $35 \mathrm{~cm}^{-1}\left(45 \mathrm{~cm}^{-1}\right)$ in $\mathrm{D}_{2} \mathrm{O}\left(\mathrm{H}_{2} \mathrm{O}\right)$. Instead, the broadening of the anharmonic simulation is fully obtained $a b$ initio from phonon-phonon scattering.

Both the dielectric function of Fig. 9 and the Raman scattering spectra in Fig. 8 confirm the importance of anharmonicity. They further demonstrate how it is fundamental to reproduce the experimental results and provide the SSCHA (and its time-dependent extension) as the best tool for ice simulation. 


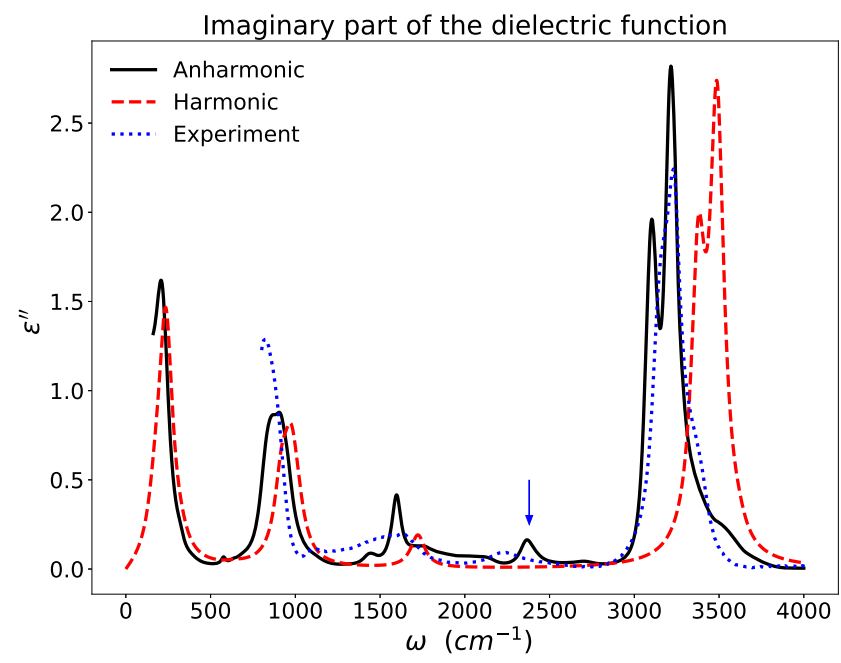

FIG. 9. Imaginary part of the dielectric function of $\mathrm{H}_{2} \mathrm{O}$ ice at $\mathrm{T}=200 \mathrm{~K}$. The trace of the dielectric tensor as in Eq. (C6) is reported to take into consideration all the possible orientation of the crystal. The results within the harmonic approximation (red dashed curve) and the anharmonic phonons (black solid line), computed in SSCHA framework with the inclusion of the bubble term of Eq. (A7), are compared with the experiment ${ }^{38}$ (blue dotted line). The blue arrow indicates a combination mode. We employed an artificial broadening of $50 \mathrm{~cm}^{-1}$ in the harmonic spectrum. Instead, the broadening of the anharmonic simulation is fully obtained $a b$ initio from phonon-phonon scattering.

\section{CONCLUSIONS}

Exploring the thermodynamic structural and vibrational properties of ice XI (hydrogen-ordered counterpart of ordinary ice $I_{h}$ ), we further elucidated the importance of quantum anharmonic effects. The anomalous strong temperature dependence of the bulk modulus, $20 \%$ variation from 0 to $300 \mathrm{~K}$, is fully explained by thermal and quantum anharmonic fluctuations, revealing the combined effect of the vibrations (64\%) and the thermal expansion (36\%).

We highlight the inaccuracy of the quasi-harmonic approximation in reproducing the anomalous VIE. Only an anharmonic treatment of quantum nuclear motion enables the reproduction of the experimental results. In particular, we proved how the negative VIE originates from a nonmonotonous volume expansion due to quantum fluctuations. If we increase the mass of the hydrogen isotopes, the volume first expands, saturating slightly above the tritium mass, and then contracts to the classical value. This means that the VIE is due to a strongly nonlinear regime of quantum fluctuations in ice, which commonly employed approximate theories (such as the QHA) do not grasp. Notably, also oxygen is in a strong quantum mechanical regime, being responsible for a $2 \%$ volume reduction in the classical limit.

We observe an anharmonic renormalization of $8 \%-10 \%$ in the bending and stretching modes that grants a good prediction of Raman scattering spectra and the dielectric function of $\mathrm{H}_{2} \mathrm{O}$ and $\mathrm{D}_{2} \mathrm{O}$ ice XI.

For the first time, the low-energy range of phonon dispersion of deuterated ice at $\mathrm{T}=140 \mathrm{~K}$ is excellently reproduced by the anharmonic renormalized phonons and correct treatment of the electron exchange and correlation. This result paves the way for the study of thermal transport from first-principles and the simulation of ice under pressure, where acoustic phonons are the only modes detectable.

Our simulations deciphered the microscopic origin of many anomalous properties of ice, proving how anharmonicity and quantum fluctuations of ions are a mandatory ingredient to reproduce the thermodynamic structural and vibrational properties of ice.

\section{ACKNOWLEDGMENTS}

The authors acknowledge the CINECA award under the ISCRA initiative for the availability of high performance computing resources and support.

\section{AUTHOR DECLARATIONS \\ Conflict of Interest}

We declare that we have no conflicts of interest to disclose.

\section{DATA AVAILABILITY}

The data that support the findings of this study are available from the corresponding author upon reasonable request.

\section{APPENDIX A: THEORETICAL METHODS}

In Sec. III C, we mentioned that the SSCHA dynamical matrix is not suited to describe real phonons. ${ }^{60,62,82}$ This happens because it is positive-definite by construction so that, for example, phonons instabilities cannot occur. The eigenvalues of the Hessian matrix of Eq. (A1) are the response to a static external perturbation and describe the stability of the structure with respect to a spontaneous symmetry breaking,

$$
\boldsymbol{D}^{(\mathrm{F})}=\frac{1}{\sqrt{\mathbf{M}}} \cdot \frac{\partial^{2} \mathcal{F}}{\partial \mathcal{R} \partial \mathcal{R}} \cdot \frac{1}{\sqrt{\mathbf{M}}} .
$$

Here, $\mathcal{F}$ is a short-hand notation for the SSCHA free energy in Eq. (3). The Hessian matrix can be written in terms of the third- and fourth-order force constant matrices, $\stackrel{(3)}{\boldsymbol{\Phi}}, \underset{(\mathbf{\Phi}}{\boldsymbol{\Phi}}$, as in Refs. 60 and 82, where

$$
\stackrel{(\mathrm{n})}{\boldsymbol{\Phi}}=\langle\underbrace{\frac{\partial^{n} \mathcal{F}}{\partial \mathcal{R} \ldots \partial \mathcal{R}}}_{\mathrm{n}}\rangle_{\tilde{\rho}_{\mathcal{R}, \Phi}} .
$$

In this work, we use the lowest order correction in the Hessian matrix, the one containing the third-order force constant matrix, after checking that the fourth-order contribution is negligible. In these conditions, the free energy Hessian can be approximated as in the following equation:

$$
D^{(\mathrm{F})} \simeq D^{(\mathrm{S})}+\stackrel{(3)}{D}: \Lambda[0]: \stackrel{(3)}{D},
$$

where $\boldsymbol{D}^{(\mathrm{S})}$ is the SSCHA dynamical matrix and the other term is called the bubble correction (as, at lowest order perturbation theory, it gives rise to the bubble diagram)..$\stackrel{60}{D}$ is the $\mathrm{n}$-th order 
force constant matrix divided by the square root of the masses (the superscript ${ }^{(2)}$ is neglected for the second order SSCHA dynamical matrix),

$$
\stackrel{(\mathrm{n})}{D}=\underbrace{\frac{\stackrel{(\mathrm{n})}{\Phi}}{\sqrt{M} \ldots \sqrt{M}}}_{\mathrm{n}} .
$$

$\Lambda[0]$ is the zero frequency value of the fourth-order tensor that depends only on the eigenvalues $\omega_{\mu}$ and eigenvectors $\boldsymbol{\epsilon}_{\mu}$ of the SCHA auxiliary dynamical matrix $D^{(\mathrm{S})}$,

$$
\begin{aligned}
(\Lambda[z])^{a b c d} & =\sum_{\mu v=1}^{3 N} \mathscr{F}\left(z, \omega_{\mu}, \omega_{v}\right) \epsilon_{\nu}^{a} \epsilon_{\mu}^{b} \epsilon_{v}^{c} \epsilon_{\mu}^{d}, \\
\mathscr{F}\left(z, \omega_{\mu}, \omega_{v}\right)= & -\frac{1}{4 \omega_{\mu} \omega_{v}} \frac{\left(\omega_{\mu}+\omega_{v}\right)\left(n_{\mu}+n_{v}+1\right)}{\left(\omega_{\mu}+\omega_{v}\right)^{2}-z^{2}} \\
& +\frac{1}{4 \omega_{\mu} \omega_{v}} \frac{\left(\omega_{\mu}-\omega_{v}\right)\left(n_{\mu}-n_{v}\right)}{\left(\omega_{\mu}-\omega_{v}\right)^{2}-z^{2}} .
\end{aligned}
$$

The $a, b, c$, and $d$ indices run over the atoms in the supercell and the Cartesian coordinates.

The $\Lambda$ tensor describes the propagation of the $\mu \nu$ phonon modes, whose interaction can give rise to combination modes, as seen in Fig. 7. By restricting the sum in Eq. (A4), it is possible to isolate the contribution of few selected modes to the free energy Hessian.

The eigenvalues of the Hessian matrix can be used to approximate the real phonons in the low-energy regime, close to $\mathrm{z}=0$, as in Fig. 6. However, physical phonons, those observed by experimental probes like inelastic scattering and vibrational spectroscopy, must be computed from the dynamical interacting Green function. Within the SSCHA framework, the dynamical Green function $\boldsymbol{G}(z)$ for the displacement normalized to the masses, $\sqrt{\boldsymbol{M}}(\boldsymbol{R}-\mathcal{R})$, in component free notation, is ${ }^{60,62,82}$

$$
\boldsymbol{G}^{-1}(z)=z^{2} \mathbb{1}-\left(\boldsymbol{D}^{(\mathrm{S})}-\boldsymbol{\Pi}(z)\right) .
$$

$D^{(S)}$ is the SSCHA dynamical matrix at equilibrium. The full expression for the SSCHA self-energy $\Pi(z)$ can be found in Refs. 60 and 82 .

In this work, as for the free energy Hessian, we decide to keep the lowest order of the self-energy correction, the bubble $\stackrel{(\mathrm{B})}{\Pi}(z)$,

$$
\Pi(z) \simeq \stackrel{(B)}{\Pi}(z)=\stackrel{(3)}{D}: \Lambda(z): \stackrel{(3)}{D}
$$

The real phonons associated are the poles of the dynamical Green function (see Ref. 82, Sec. 4.3, for further details on the computation of the poles $\Omega_{\mu}$ and linewidths $\Gamma_{\mu}$ ).

\section{APPENDIX B: COMPUTATIONAL DETAILS}

To simulate the thermodynamic properties, we computed the harmonic free energy (relaxing the atomic position at fixed cell) at
120 volumes. The free energy is fitted with the Vinet equation of state $(\mathrm{EOS})^{78}$ to obtain the equilibrium volume and the bulk modulus for any temperature as in Eqs. (4) and (5).

In contrast, for the SSCHA, we computed the pressure as a function of temperature for six volumes. For each volume, we relaxed the atomic positions accounting for quantum and thermal anharmonic effects. We employed ensembles with as many as 100000 configurations in the converged supercell to reduce the statistical noise. We evaluated the equilibrium volume and the bulk modulus fitting the $P(\Omega)$ curve with the Vinet EOS at each temperature.

For QHA simulations, we sampled phonons in a $14 \times 14 \times 14$ mesh of the Brillouin zone; for the SSCHA ones, we employed a $3 \times 3$ $\times 2$ supercell. The convergence tests are reported in Appendix D.

The spectral properties are computed from the dynamical onephonon interacting Green function. The self-energy is approximated as in Eq. (A7), and it is computed integrating on a $14 \times 14$ $\times 14 \boldsymbol{k}$-grid in the reciprocal space. ${ }^{73}$ Furthermore, a smearing factor $\delta_{s e}$ is introduced to obtain converged results in the computation,

$$
\Pi(z) \simeq \stackrel{(\mathrm{B})}{\Pi}(z)=\stackrel{(\mathrm{B})}{\Pi}\left(z+i \delta_{s e}\right) .
$$

Convergence is achieved for $\delta_{s e}=45 \mathrm{~cm}^{-1}$. The same value holds for $\mathrm{H}_{2} \mathrm{O}$ and $\mathrm{D}_{2} \mathrm{O}$ ice. Consequently, all the SSCHA spectral functions are computed with those values of smearing and integration of $\boldsymbol{k}$-grid. Finite linewidths in the DOS and harmonic model are for presentation purposes only.

\section{APPENDIX C: RAMAN AND INFRARED}

The Raman spectrum is proportional to the polarizability correlation function $\langle\alpha(t) \alpha(0)\rangle$, where

$$
\alpha_{a b}(t)=\sum_{c=1}^{3 N} A_{a b c} u_{c}(t)
$$

The Raman tensor $A_{a b c}$ is computed $a b$ initio with Quantum ESPRESSO $^{79}$ in the SSCHA average centroid positions within the local-density approximation (LDA). The electronic wave functions were expanded in plane waves up to a cutoff of $120 \mathrm{Ry}$ (960 Ry for the electronic density), with a uniform grid of $4 \times 4 \times 4$ for the Brillouin zone integrals. Here, $\mathrm{a}$ and $\mathrm{b}$ are the Cartesian indices and $\mathrm{c}$ is a super-index running over the Cartesian coordinates and the atoms in the supercell. We keep this notation in the following.

The intensity of the Raman signal has been evaluated as

$$
I^{\text {Raman }}(\omega) \propto\left(\omega-\omega_{L}\right)^{4} \sum_{a, b=1}^{3 N} \frac{A_{a}^{\prime} A_{b}^{\prime}}{\sqrt{M_{a} M_{b}}} \operatorname{Im} G_{a b}(\omega),
$$

where $\omega_{L}$ is the frequency of the laser and $A^{\prime}$ accounts for the polarization of the incident $\left(\epsilon^{\text {in }}\right)$ and scattered $\left(\epsilon^{\text {out }}\right)$ light, ${ }^{37,41,42}$

$$
A^{\prime}=\epsilon^{\text {in }} \cdot A \cdot \epsilon^{\text {out }} \text {. }
$$

In most cases, the laser frequency is much bigger than the phonon frequencies so that the approximation $\left|\omega-\omega_{L}\right| \simeq \omega_{L}$ holds. This is not completely true in ice, where phonons can be very 
energetic $\left(\omega \simeq 3400 \mathrm{~cm}^{-1}\right)$. Here, that difference provides a slight reshaping of the spectra, and it is safer to avoid approximations.

The physical quantity determining the infrared absorption is the dielectric function, and standard electromagnetism provides a simple relation between the dielectric tensor and the susceptibility,

$$
\epsilon(\omega)=1+4 \pi \chi^{(\mathrm{tot})}(\omega)=\epsilon^{\mathrm{el}}+4 \pi \chi^{\mathrm{ion}}(\omega) .
$$

The electronic part $\epsilon^{\mathrm{el}}$ is computed $a b$ initio. The absence of electronic transitions in the phonon energy range makes it real, and it is frequency independent, $\epsilon^{\mathrm{el}}=1.65$. The ionic susceptibility of Eq. (C3) is the Fourier transform of the dipole-dipole correlation function,

$$
\chi_{a b}^{\text {ion }}(\omega)=\int d t e^{-i \omega t}\left\langle M_{a}(t) M_{b}(0)\right\rangle,
$$

where $M_{a}(t)=|e| \sum_{b=1}^{3 N} Z_{a b} u_{b}(t)$; the effective charges $Z$ are computed $a b$ initio in Quantum ESPRESSO ${ }^{79}$ in LDA. The electronic wave functions were expanded in plane waves up to a cutoff of $120 \mathrm{Ry}$ (960 Ry for the electronic density), with a uniform grid of $4 \times 4 \times 4$ for the Brillouin zone integrals.

We computed the dielectric function as

$$
\epsilon_{\alpha \beta}(\omega)=\epsilon_{\alpha \beta}^{\mathrm{el}}+4 \pi|e|^{2} \sum_{a b} \frac{Z_{\alpha a} Z_{\beta b}}{\sqrt{M_{a} M_{b}}} G_{a b}(\omega)
$$

and averaged over all possible orientation of the crystal,

$$
\epsilon(\omega)=\frac{1}{3} \sum_{\alpha=1}^{3} \epsilon_{\alpha \alpha}(\omega) .
$$

\section{APPENDIX D: CONVERGENCE}

Equations (1) and (3) depend on the number $N_{q}$ of $q$ points in the Brillouin zone. We employ the supercell method that consists in replicating an integer number of times $N_{1} \times N_{2} \times N_{3}$ the unit cell along the three Cartesian directions and imposing periodic boundary conditions. The unit cell of ice XI has an orthorhombic structure with the $C m c 2_{1}$ space group and contains four water molecules. In Sec. III A, we pointed out that the thermodynamic properties in the QHA are derived from the free energy, while in the SSCHA the pressure has been used, thanks to the availability of a handy analytical formula.

Consequently, the converged supercells are chosen by looking at the free energy in the QHA and at the pressure in the SSCHA. We analyzed the convergence for the two extremal temperatures, $\mathrm{T}=0 \mathrm{~K}$ and $\mathrm{T}=300 \mathrm{~K}$, in Fig. 10. As a general trend, we notice that thermal fluctuations slow down the convergence of both the free energy and the pressure. The limited computational cost of the QHA does not place any limitation on the mesh to use.

Moreover, we have the possibility to interpolate the dynamical matrix to a finer mesh, as it has been done for the two bigger meshes, namely, the $10 \times 10 \times 10$ and the $14 \times 14 \times 14$. Hence, by looking at Fig. 10(b), we decided to use the biggest grid we analyzed.
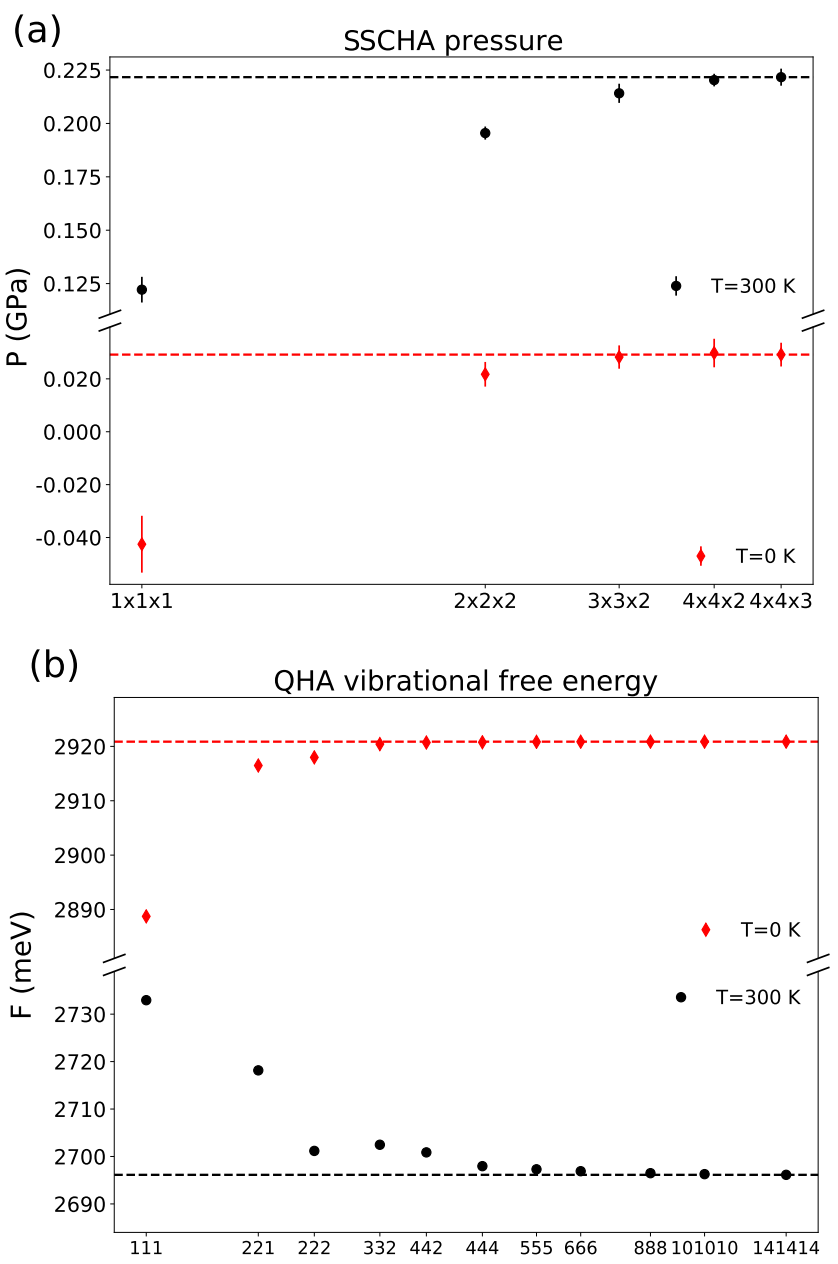

FIG. 10. Convergence tests in the QHA and SSCHA. (a) Pressure within the SSCHA framework computed as in Ref. 61 as a function of supercell dimension for $\mathrm{T}=0 \mathrm{~K}$ and $\mathrm{T}=300 \mathrm{~K}$. (b) Vibrational term of the QHA free energy in Eq. (1) as a function of supercell dimension for $\mathrm{T}=0 \mathrm{~K}$ and $\mathrm{T}=300 \mathrm{~K}$. Here, we use the short hard notation $N_{1} N_{2} N_{3}$ to indicate the $N_{1} \times N_{2} \times N_{3}$ supercell. The dashed lines in panels (a) and (b) are the converged results for the pressure or the free energy at the given temperature.

Conversely, the challenging computational cost of the SSCHA prevents the use of a big supercell. Figure 10(a) reveals as the $3 \times 3 \times 2$ supercell grants at most $3 \%$ error with respect to the converged mesh at high temperature. We are satisfied with this accuracy level. We compute the thermodynamic properties in this mesh. Instead, to compute the phonon dispersion of Sec. III C, a $4 \times 4 \times 4$ supercell has been used.

\section{APPENDIX E: THERMODYNAMIC PROPERTIES}

While the effect of anharmonicity on the covalent bond length is counter intuitive as reported in Sec. III A (Fig. 3), the hydrogen bond length follows the expected trend (Fig. 11). Anharmonicity shrinks the H-bond length since 


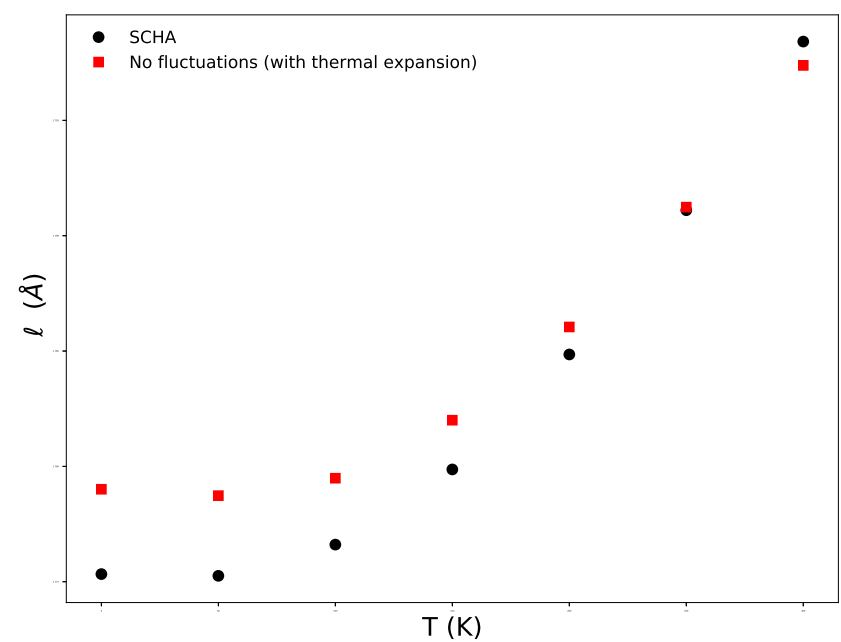

FIG. 11. Temperature dependence of the hydrogen bond length. The SSCHA bond length (black circles) is compared with the classical result (red squares), computed from the minimization of the BO energy $V\left(\mathcal{R},\left\{\vec{a}_{i}\right\}\right)$, where the effect of the thermal expansion is introduced by fixing the volume to be the SSCHA equilibrium one at each temperature, in order to point out the contribution of thermal and quantum fluctuations.

its widening upon heating explains thoroughly the volume expansion.

The bulk modulus is computed from Eq. (5). The free energy consists in a static and a vibrational term as in Eq. (1), so the same is for the bulk modulus. In the QHA picture, the static part and the vibrational part are in the following equations:

$$
\begin{gathered}
B_{\text {stat }}(T)=\left.\Omega_{e q}(T) \frac{\partial^{2} V\left(\mathcal{R},\left\{\vec{a}_{i}\right\}\right)}{\partial \Omega^{2}}\right|_{\Omega_{e q(T)}}, \\
B_{v i b}(T)=\left.\Omega_{e q}(T) \frac{\partial^{2} F_{v i b}\left(\mathcal{R},\left\{\vec{a}_{i}\right\}\right)}{\partial \Omega^{2}}\right|_{\Omega_{e q(T)}} .
\end{gathered}
$$

The division of the bulk modulus into its two contributions makes possible to individuate the origin of its strong temperature dependence observed in Fig. 2.

Figure 12(a) shows the static bulk modulus, where fluctuations are neglected. We computed the curves at the QHA and SSCHA equilibrium volumes, in order to introduce the effect of thermal expansion. The continuous line is the classic result, where quantum and thermal effects are not included. The vibrational contribution of Eq. (E1b), for the QHA and SSCHA volumes, is reported in Fig. 12(b). It is always negative, meaning that it would increase the volume under compression.

Both the static modulus and the vibrational bulk modulus have a non-negligible temperature dependence, contributing to the total one for the $64 \%$ and $36 \%$, respectively.

Moreover, the effect of different equilibrium volumes is almost temperature independent in the vibrational term, being unable to explain the different thermal behavior of the two theories, which can instead be addressed partially to the effect of volumes in the static bulk modulus as evident in Fig. 12(a). (a)

(b)
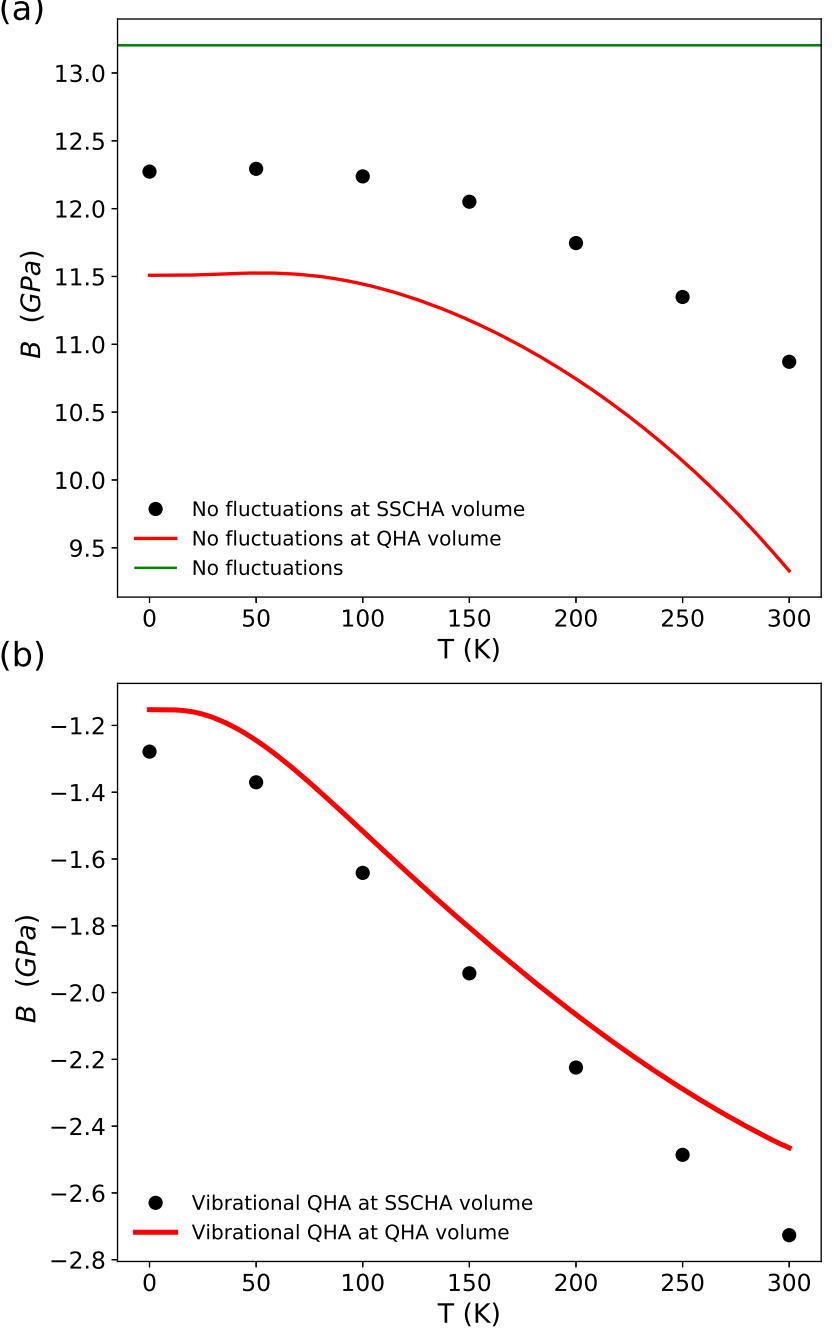

FIG. 12. Analysis of the different contributions to the bulk modulus. (a) The static QHA bulk modulus is computed at the QHA equilibrium volumes (solid red line) and at the SSCHA equilibrium volumes (black circles). The green solid line is the classical bulk modulus where fluctuations are neglected. (b) Vibrational QHA bulk modulus computed at the SSCHA (black circles) and QHA (red solid line) equilibrium volumes.

\section{APPENDIX F: DISPERSION}

Real phonon dispersion is calculated from the dynamical interacting Green function as in Appendix A. Here, we show the density of states (DOS) and dispersion for $\mathrm{H}_{2} \mathrm{O}$ ice at $\mathrm{T}=200 \mathrm{~K}$ and $\mathrm{D}_{2} \mathrm{O}$ ice at $\mathrm{T}=140 \mathrm{~K}$ and $\mathrm{P}=0.05 \mathrm{GPa}$, comparing the harmonic phonons and the SSCHA auxiliary phonons in Fig. 13.

The harmonic dynamical matrix is computed with a $5 \times 5 \times 5 \boldsymbol{q}$ mesh, while for the SSCHA, we employed the $4 \times 4 \times 4$ supercell.

As a molecular crystal, the phonon branches are well separated in translational modes, librations, narrow bending, and stretching from low to high energy. In Fig. 13(a), we report the hydrogen, and in Fig. 13(b), we report the deuterium. 
(a)

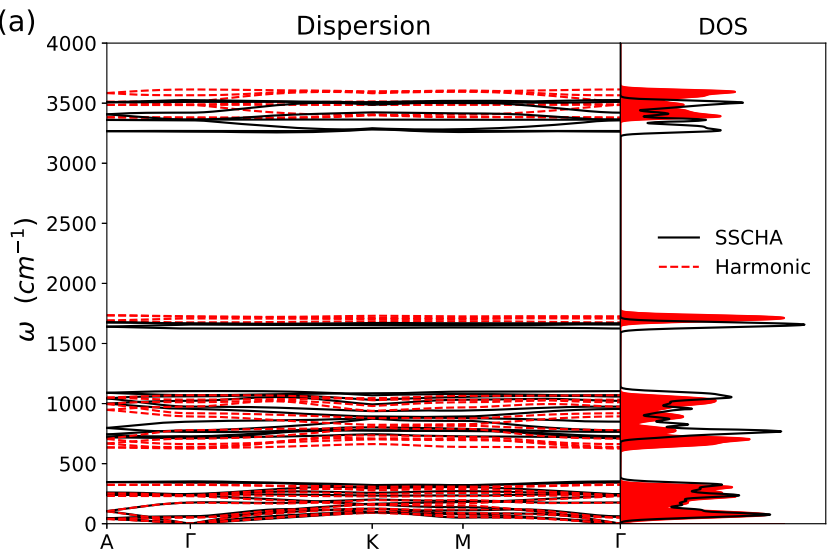

(b)

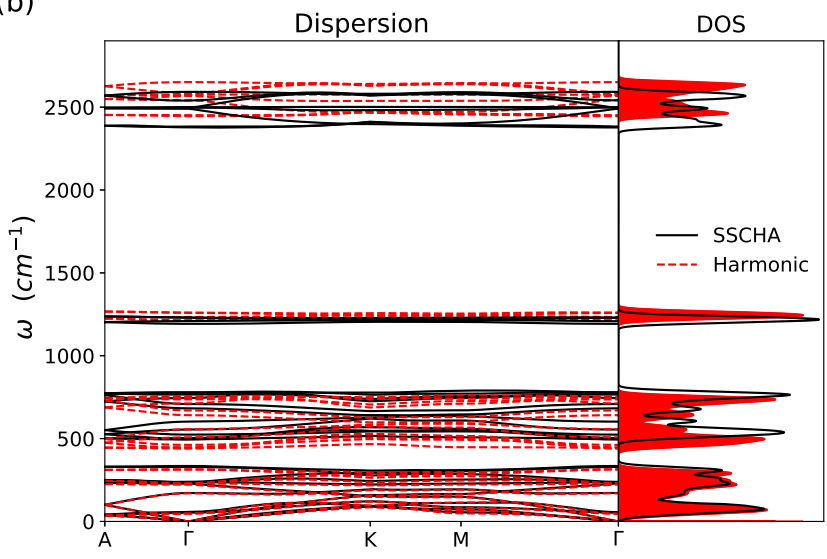

FIG. 13. Phonon dispersion and density of states. (a) $\mathrm{H}_{2} \mathrm{O}$ ice $\mathrm{XI}$ at $\mathrm{T}=200 \mathrm{~K}$ at ambient pressure. (b) $\mathrm{D}_{2} \mathrm{O}$ ice XI at $\mathrm{T}=140 \mathrm{~K}$ and $\mathrm{P}=0.05 \mathrm{GPa}$. In both panels (a) and (b), the harmonic results (red dashed line) are compared with the dispersion and DOS in the SSCHA framework (black solid lines).

In the SSCHA, the harmonic translational and rotational modes suffer a blue shift of the order of $4.2 \%(3.5 \%)$ and $8.2 \%(7.5 \%)$, respectively, for $\mathrm{H}_{2} \mathrm{O}\left(\mathrm{D}_{2} \mathrm{O}\right)$ ice. Instead, anharmonicity reduces the frequencies of the most energetic modes. Both the harmonic bending and stretching band are red-shifted of about $3.1 \%(2.2 \%-2.5 \%)$ in $\mathrm{H}_{2} \mathrm{O}\left(\mathrm{D}_{2} \mathrm{O}\right)$ ice.

Acoustic modes play a major role in thermal transport. We compared the simulated phonon dispersion of deuterated ice at $\mathrm{T}=140 \mathrm{~K}$ and $\mathrm{P}=0.05 \mathrm{GPa}$ in Fig. 6 with the experiment. For a correct comparison, real phonons are needed. In the low-energy regime, we can use the static approximation of the self-energy. ${ }^{60}$ We consider the lowest order self-energy correction, the bubble of Eq. (A2). This approximation is reasonable and routinely employed in many systems with hydrogen. ${ }^{80,81}$ In the present case, we checked the approximation against the exact static self-energy, where phonon energies change less than $1 \mathrm{~cm}^{-1}$.

While the harmonic phonons can be computed without effort in the supercell described above, the computational cost of the bubble term is huge, and it is not possible to perform the calculations in the same supercell used for the SSCHA dynamical matrix $\boldsymbol{D}^{(\mathrm{S})}$. To overcome this problem, we first computed the bubble correction in a smaller supercell, namely, a $3 \times 3 \times 2$, and then, we interpolated it to a finer supercell to obtain the Hessian matrix,

$$
\boldsymbol{D}_{4 \times 4 \times 4}^{(\mathrm{F})}=\boldsymbol{D}_{4 \times 4 \times 4}^{(\mathrm{S})}+\left[\boldsymbol{D}_{3 \times 3 \times 2}^{(\mathrm{F})}-\boldsymbol{D}_{3 \times 3 \times 2}^{(\mathrm{S})}\right]^{4 \times 4 \times 4} .
$$

In Fig. 6, we restricted the original path of the experiment (the same as in Fig. 13) to the $\Gamma$-A direction. We include the $\Gamma$-K path dispersion in Fig. 14. In ice XI, we have two inequivalent $\Gamma$-K paths originated by the presence of the hydrogen sublattice that overturns the perfect equivalence we would obtain in the presence of oxygens alone.

The projection of the first Brillouin zone of ice XI in the plane is reported in the inset of Fig. 14(a). We show the planar reciprocal

(a)

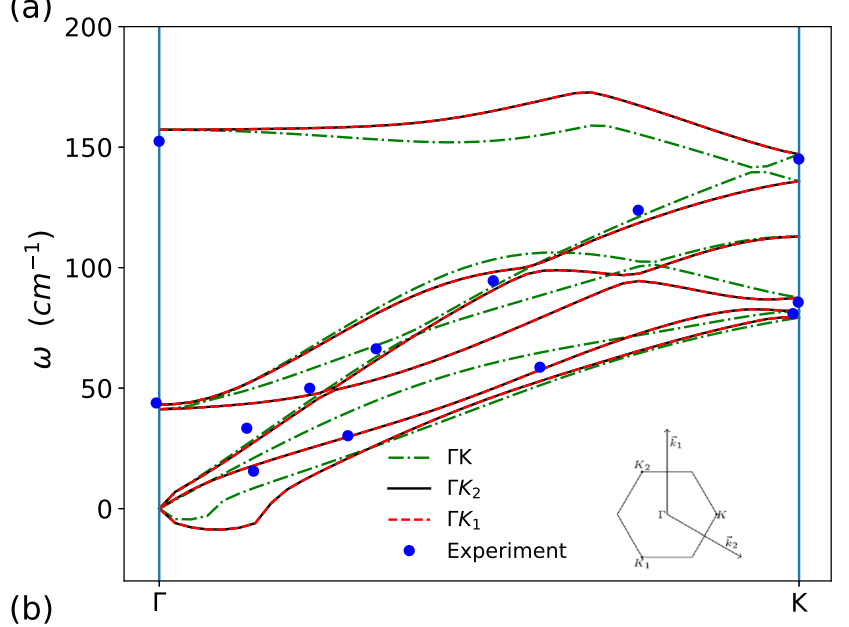

(b)

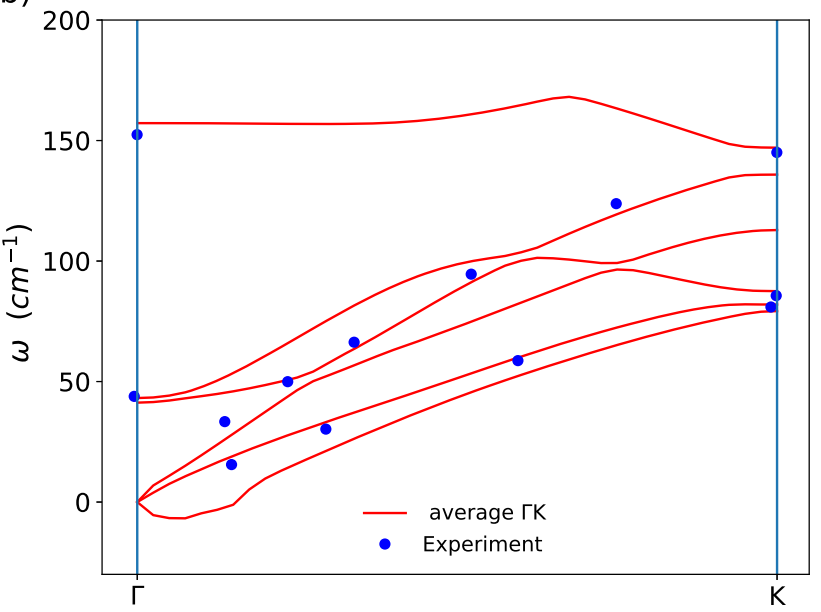

FIG. 14. Phonon dispersion in the SSCHA framework with the inclusion of the bubble term in the self-energy correction in the static limit for $\mathrm{D}_{2} \mathrm{O}$ ice at $\mathrm{T}=140 \mathrm{~K}$ and $\mathrm{P}=0.05 \mathrm{GPa}$. (a) Phonon dispersion along the three nonequivalent $\Gamma K$ directions compared to the experimental measurement (blue circles). ${ }^{55}$ The three $\mathrm{K}$ points and the projection in the plane of the first Brillouin Zone are shown in the inset. (b) Comparison of the experiment with the average of the phonon dispersion along the three $\Gamma K$ directions (red solid lines). 
vectors, $\left\{\vec{b}_{1}, \vec{b}_{2}\right\}$, and the three K points. Notably, the three K points are actually equivalent (the phonon frequencies are the same), but the modes along the path connecting $\Gamma$ and $\mathrm{K}$ are not.

We average the three dispersion and compared the average with the experiment in Fig. 14(b). The matching between theory and experiment is very good.

\section{APPENDIX G: COMBINATION MODES}

Figure 13 reveals the presence features in the spectral function that are absent in the phonon DOS. As discussed in Sec. III D, this hints the occurrence of combination modes originated by phonon-phonon interactions.

We can dissect the interaction between phonon bands to unveil which modes originate these satellite peaks in the spectral function (see Appendix A for further details).

We report the results for $\mathrm{H}_{2} \mathrm{O}$ ice at $\mathrm{T}=200 \mathrm{~K}$, but the same also holds in deuterated ice (whose spectral function and DOS at $\mathrm{T}=140 \mathrm{~K}$ and $\mathrm{P}=0.05 \mathrm{GPa}$ are in Fig. 15). We show in Fig. 16 that the first combination mode, occurring for $\omega \in[2250,2800] \mathrm{cm}^{-1}$, is originated from the interaction between the libration and bending bands, as the peak appears only if we account for their reciprocal interaction. If we account only for librational or bending modes in the calculations, the peak vanishes. The residual mismatch in the low-frequency tail with the full spectral function reveals a non-negligible contribution of the other phonon branches (mainly translations).

The overtone at twice the stretching frequency is analyzed in Fig. 17. The perfect matching between the full spectral function and the same computed only considering stretching modes unveils how this peak is entirely generated by stretching modes interacting with themselves, without a significative contribution of other phonon branches. It is, in fact, the overtone of the stretching modes.

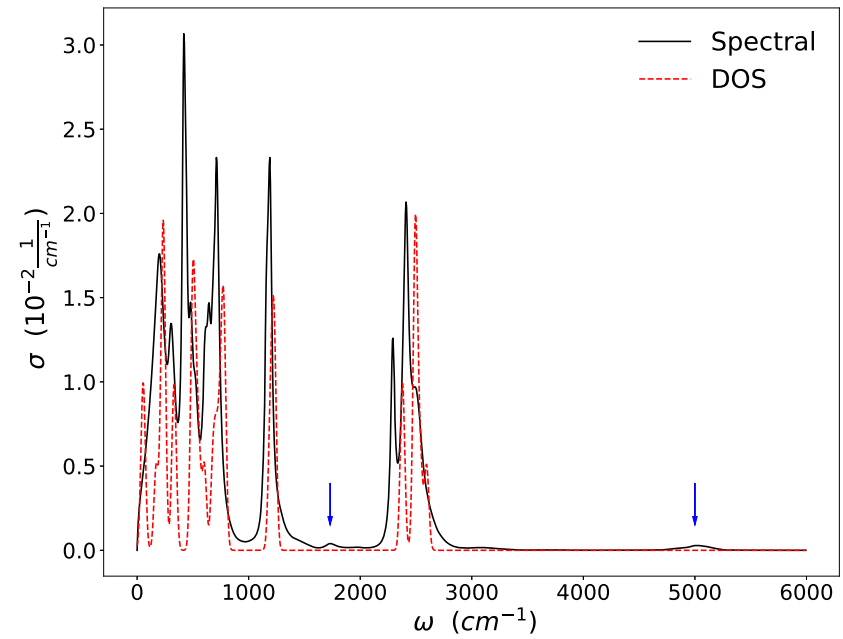

FIG. 15. Comparison of the density of states and spectral function at $\Gamma . D_{2} \mathrm{O}$ ice at $\mathrm{T}=140 \mathrm{~K}$ and $\mathrm{P}=0.05 \mathrm{GPa}$. The spectral function (black solid line) and DOS (red dashed line) are shown. The DOS is computed by using the SSCHA dynamical matrix at equilibrium without the inclusion of the self-energy term and by adding an artificial smearing factor of $20 \mathrm{~cm}^{-1}$. The blue arrows indicate the combination modes and anharmonic overtones.

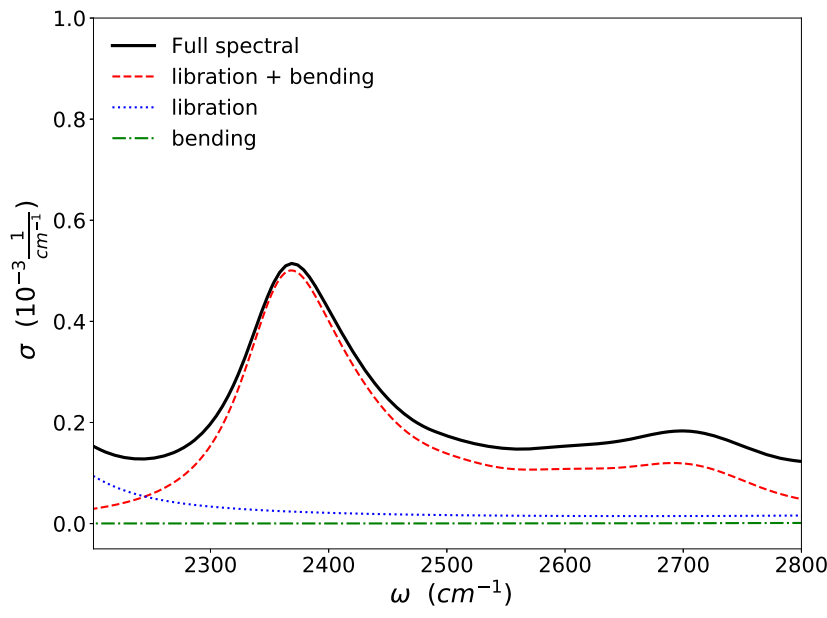

FIG. 16. Spectral function of $\mathrm{H}_{2} \mathrm{O}$ ice at $\mathrm{T}=200 \mathrm{~K}$ in the energy range of the first combination mode at $\Gamma$. A comparison between the full spectral function (solid black line) and that obtained by selecting only the interaction between the librations and the bending band (red dashed line) is provided. Spectral functions obtained through the interaction of bending band with themselves (green dotted-dashed line) and librations with themselves (blue dotted line) are shown too.

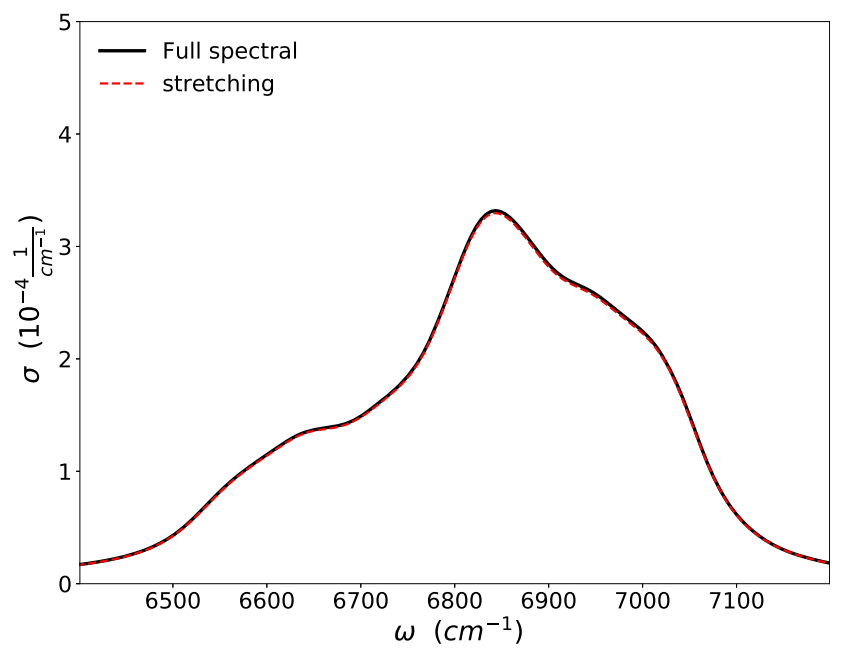

FIG. 17. Spectral function of $\mathrm{H}_{2} \mathrm{O}$ ice at $\mathrm{T}=200 \mathrm{~K}$ in the energy range of the second combination mode at $\Gamma$. The full spectral function (solid black line) is compared to that obtained by selecting only the interaction of the stretching band with itself (red dashed line).

\section{REFERENCES}

${ }^{1}$ T. Bartels-Rausch, V. Bergeron, J. H. E. Cartwright, R. Escribano, J. L. Finney, H. Grothe, P. J. Gutiérrez, J. Haapala, W. F. Kuhs, J. B. C. Pettersson, S. D. Price, C. I. Sainz-Díaz, D. J. Stokes, G. Strazzulla, E. S. Thomson, H. Trinks, and N. Uras-Aytemiz, "Ice structures, patterns, and processes: A view across the icefields," Rev. Mod. Phys. 84, 885-944 (2012).

${ }^{2}$ A. D. Fortes, I. G. Wood, D. Grigoriev, M. Alfredsson, S. Kipfstuhl, K. S. Knight, and R. I. Smith, "No evidence for large-scale proton ordering in Antarctic ice from powder neutron diffraction," J. Chem. Phys. 120, 11376-11379 (2004). 
${ }^{3}$ A. J. Leadbetter, R. C. Ward, J. W. Clark, P. A. Tucker, T. Matsuo, and H. Suga, "The equilibrium low-temperature structure of ice," J. Chem. Phys. 82, 424-428 (1985).

${ }^{4}$ C. Lobban, J. L. Finney, and W. F. Kuhs, "The structure of a new phase of ice," Nature 391, 268-270 (1998).

${ }^{5}$ B. Kamb, “Ice. II. A proton-ordered form of ice," Acta Crystallogr. 17, 1437-1449 (1964).

${ }^{6}$ B. Kamb, A. Prakash, and C. Knobler, "Structure of ice. V," Acta Crystallogr. 22, 706-715 (1967).

${ }^{7}$ B. Kamb and A. Prakash, "Structure of ice III," Acta Crystallogr., Sect. B: Struct. Crystallogr. Cryst. Chem. 24, 1317-1327 (1968).

${ }^{8}$ B. Kamb and B. L. Davis, "Ice VII, the densest form of ice," Proc. Natl. Acad. Sci. U. S. A. 52, 1433-1439 (1964).

${ }^{9}$ H. Engelhardt and B. Kamb, "Structure of ice IV, a metastable high-pressure phase," J. Chem. Phys. 75, 5887-5899 (1981).

${ }^{10} \mathrm{~L}$. G. Dowell and A. P. Rinfret, "Low-temperature forms of ice as studied by x-ray diffraction," Nature 188, 1144-1148 (1960).

${ }^{11}$ J. D. Bernal and R. H. Fowler, "A theory of water and ionic solution, with particular reference to hydrogen and hydroxyl ions," J. Chem. Phys. 1, 515-548 (1933).

${ }^{12}$ Y. Tajima, T. Matsuo, and H. Suga, "Phase transition in $\mathrm{KOH}$-doped hexagonal ice," Nature 299, 810-812 (1982).

${ }^{13} \mathrm{Y}$. Tajima, T. Matsuo, and H. Suga, "Calorimetric study of phase transition in hexagonal ice doped with alkali hydroxides," J. Phys. Chem. Solids 45, 1135-1144 (1984).

${ }^{14} \mathrm{~S}$. Kwada, "Acceleration of dielectric relaxation by $\mathrm{KOH}$-doping and phase transition in ice Ih," J. Phys. Chem. Solids 50, 1177-1184 (1989).

${ }^{15}$ M. Marchi, J. S. Tse, and M. L. Klein, "Lattice vibrations and infrared absorption of ice Ih," J. Chem. Phys. 85, 2414-2418 (1986).

${ }^{16} \mathrm{~J}$. S. Tse, M. L. Klein, and I. R. McDonald, "Lattice vibrations of ices Ih, VIII, and IX," J. Chem. Phys. 81, 6124-6129 (1984).

${ }^{17}$ P. Bosi, R. Tubino, and G. Zerbi, "On the problem of the vibrational spectrum and structure of ice Ih: Lattice dynamical calculations," J. Chem. Phys. 59, 4578-4586 (1973).

${ }^{18} \mathrm{~K}$. Röttger, A. Endriss, J. Ihringer, S. Doyle, and W. F. Kuhs, "Lattice constants and thermal expansion of $\mathrm{H}_{2} \mathrm{O}$ and $\mathrm{D}_{2} \mathrm{O}$ ice $\mathrm{I} h$ between 10 and 265 K," Acta Crystallogr., Sect. B: Struct. Sci. 50, 644-648 (1994).

${ }^{19}$ A. D. Fortes, "Accurate and precise lattice parameters of $\mathrm{H}_{2} \mathrm{O}$ and $\mathrm{D}_{2} \mathrm{O}$ ice $\mathrm{I} h$ between 1.6 and $270 \mathrm{~K}$ from high-resolution time-of-flight neutron powder diffraction data," Acta Crystallogr., Sect. B: Struct. Sci., Cryst. Eng. Mater. 74, 196-216 (2018).

${ }^{20} \mathrm{H}$. Tanaka, "Thermodynamic stability and negative thermal expansion of hexagonal and cubic ices," J. Chem. Phys. 108, 4887-4893 (1998).

${ }^{21}$ K. Umemoto, E. Sugimura, S. de Gironcoli, Y. Nakajima, K. Hirose, Y. Ohishi, and R. M. Wentzcovitch, "Nature of the volume isotope effect in ice," Phys. Rev. Lett. 115, 173005 (2015).

${ }^{22}$ B. Pamuk, J. M. Soler, R. Ramírez, C. P. Herrero, P. W. Stephens, P. B. Allen, and M. V. Fernández-Serra, "Anomalous nuclear quantum effects in ice," Phys. Rev. Lett. 108, 193003 (2012).

${ }^{23}$ M. A. Salim, S. Y. Willow, and S. Hirata, "Ice Ih anomalies: Thermal contraction, anomalous volume isotope effect, and pressure-induced amorphization," J. Chem. Phys. 144, 204503 (2016).

${ }^{24}$ R. Ramírez, N. Neuerburg, M.-V. Fernández-Serra, and C. P. Herrero, "Quasiharmonic approximation of thermodynamic properties of ice Ih, II, and III," J. Chem. Phys. 137, 044502 (2012).

${ }^{25}$ C. P. Herrero and R. Ramírez, "Isotope effects in ice Ih: A path-integral simulation," J. Chem. Phys. 134, 094510 (2011).

${ }^{26}$ M. Fernandez-Serra, B. Pamuk, and P. B. Allen, "Nuclear quantum effects in different ice phases," in APS March Meeting Abstracts, APS Meeting Abstracts (APS, 2016), Vol. 2016, p. K47.006.

${ }^{27}$ J. Wang, G. Román-Pérez, J. M. Soler, E. Artacho, and M.-V. FernándezSerra, "Density, structure, and dynamics of water: The effect of van der Waals interactions," J. Chem. Phys. 134, 024516 (2011).
${ }^{28}$ B. Pamuk, P. B. Allen, and M.-V. Fernández-Serra, "Electronic and nuclear quantum effects on the ice XI/ice Ih phase transition," Phys. Rev. B 92, 134105 (2015).

${ }^{29}$ M. Fritz, J. M. Soler, and M. Fernandez-Serra, "A general optimization method applied to a vdW-DF functional for water," in APS March Meeting Abstracts, APS Meeting Abstracts (APS, 2016), Vol. 2016, p. C20.006.

${ }^{30} \mathrm{M}$. Fernandez-Serra, "van der Waals interactions in water and ice from density functional theory simulations: Improvements and challenges," in APS March Meeting Abstracts, APS Meeting Abstracts (APS, 2012), Vol. 2012, p. H6.001.

${ }^{31} \mathrm{~F}$. Li and J. L. Skinner, "Infrared and Raman line shapes for ice Ih. I. Dilute HOD in $\mathrm{H}_{2} \mathrm{O}$ and $\mathrm{D}_{2} \mathrm{O}$," J. Chem. Phys. 132, 204505 (2010).

${ }^{32} \mathrm{~F}$. Li and J. L. Skinner, "Infrared and Raman line shapes for ice Ih. II. $\mathrm{H}_{2} \mathrm{O}$ and $\mathrm{D}_{2} \mathrm{O}$," J. Chem. Phys. 133, 244504 (2010).

${ }^{33}$ M. J. Wójcik, K. Szczeponek, and S. Ikeda, "Theoretical study of the OH/OD stretching regions of the vibrational spectra of ice Ih," J. Chem. Phys. 117, 9850-9857 (2002).

${ }^{34} \mathrm{M}$. S. Bergren and S. A. Rice, "An improved analysis of the $\mathrm{OH}$ stretching region of the vibrational spectrum of ice Ih," J. Chem. Phys. 77, 583-602 (1982).

${ }^{35}$ S. A. Rice, M. S. Bergren, A. C. Belch, and G. Nielsen, "A theoretical analysis of the hydroxyl stretching spectra of ice Ih, liquid water, and amorphous solid water," J. Phys. Chem. 87, 4295-4308 (1983).

${ }^{36} \mathrm{~J}$. E. Bertie and E. Whalley, "Infrared spectra of ices Ih and Ic in the range 4000 to $350 \mathrm{~cm}^{-1}$," J. Chem. Phys. 40, 1637-1645 (1964).

${ }^{37}$ J. R. Scherer and R. G. Snyder, "Raman intensities of single crystal ice Ih," J. Chem. Phys. 67, 4794-4811 (1977).

${ }^{38}$ M. L. Clapp, D. R. Worsnop, and R. E. Miller, "Frequency-dependent optical constants of water ice obtained directly from aerosol extinction spectra," J. Phys. Chem. 99, 6317-6326 (1995).

${ }^{39}$ A. Y. Zasetsky, A. F. Khalizov, M. E. Earle, and J. J. Sloan, "Frequency dependent complex refractive indices of supercooled liquid water and ice determined from aerosol extinction spectra," J. Phys. Chem. A 109, 2760-2764 (2005).

${ }^{40}$ K. Abe, K. Ishii, M. Nakajima, H. Fukuda, and T. Shigenari, "Raman scattering at the proton ordering phase transition in ice crystal," Ferroelectrics $239,1-8$ (2000).

${ }^{41} \mathrm{~K}$. Abe and T. Shigenari, "Raman spectra of proton ordered phase XI of ICE I. Translational vibrations below $350 \mathrm{~cm}^{-1}$," J. Chem. Phys. 134, 104506 (2011).

${ }^{42} \mathrm{~T}$. Shigenari and K. Abe, "Vibrational modes of hydrogens in the proton ordered phase XI of ice: Raman spectra above $400 \mathrm{~cm}^{-1}$," J. Chem. Phys. 136, 174504 (2012).

${ }^{43}$ J.-C. Li, V. M. Nield, and S. M. Jackson, "Spectroscopic measurements of ice XI," Chem. Phys. Lett. 241, 290-294 (1995).

${ }^{44} \mathrm{M}$. Arakawa, H. Kagi, and H. Fukazawa, "Laboratory measurements of infrared absorption spectra of hydrogen-ordered ice: A step to the exploration of ice XI in space," Astrophys. J., Suppl. Ser. 184, 361-365 (2009).

${ }^{45} \mathrm{H}$. Fukazawa, S. Ikeda, and S. Mae, "Incoherent inelastic neutron scattering measurements on ice XI; the proton-ordered phase of ice Ih doped with KOH," Chem. Phys. Lett. 282, 215-218 (1998).

${ }^{46} \mathrm{H}$. Itoh, K. Kawamura, T. Hondoh, and S. Mae, "Molecular dynamics studies of proton ordering effects on lattice vibrations in ice Ih," Physica B 219-220, 469-472 (1996).

${ }^{47}$ H. Itoh, K. Kawamura, T. Hondoh, and S. Mae, "Polarized librational spectra of proton-ordered ice XI by molecular dynamics simulations," J. Chem. Phys. 109, 4894-4899 (1998).

${ }^{48}$ A. Erba, S. Casassa, R. Dovesi, L. Maschio, and C. Pisani, "Periodic density functional theory and local-MP2 study of the librational modes of ice XI," J. Chem. Phys. 130, 074505 (2009).

${ }^{49}$ M. Gług, M. Boczar, Ł. Boda, and M. J. Wójcik, "Analysis of librational modes of ice XI studied by Car-Parrinello molecular dynamics," Chem. Phys. 459, 102-111 (2015).

${ }^{50} \mathrm{R}$. Iftimie and M. E. Tuckerman, "Decomposing total IR spectra of aqueous systems into solute and solvent contributions: A computational approach using maximally localized Wannier orbitals," J. Chem. Phys. 122, 214508 (2005).

${ }^{51}$ W. Chen, M. Sharma, R. Resta, G. Galli, and R. Car, "Role of dipolar correlations in the infrared spectra of water and ice," Phys. Rev. B 77, 245114 (2008). 
${ }^{52}$ S. Klotz, T. Strässle, A. M. Saitta, G. Rousse, G. Hamel, R. J. Nelmes, J. S. Loveday, and M. Guthrie, "In situ neutron diffraction studies of high density amorphous ice under pressure," J. Phys.: Condens. Matter 17, S967-S974 (2005).

${ }^{53}$ S. Klotz, G. Hamel, J. S. Loveday, R. J. Nelmes, M. Guthrie, and A. K. Soper, "Structure of high-density amorphous ice under pressure," Phys. Rev. Lett. 89, 285502 (2002).

${ }^{54}$ S. Klotz, T. Strässle, R. J. Nelmes, J. S. Loveday, G. Hamel, G. Rousse, B. Canny, J. C. Chervin, and A. M. Saitta, "Nature of the polyamorphic transition in ice under pressure," Phys. Rev. Lett. 94, 025506 (2005).

${ }^{55}$ T. Strässle, A. M. Saitta, S. Klotz, and M. Braden, "Phonon dispersion of ice under pressure," Phys. Rev. Lett. 93, 225901 (2004).

${ }^{56}$ T. Strässle, S. Klotz, G. Hamel, M. M. Koza, and H. Schober, "Experimental evidence for a crossover between two distinct mechanisms of amorphization in ice $\mathrm{I} h$ under pressure," Phys. Rev. Lett. 99, 175501 (2007).

${ }^{57}$ J. M. Besson, S. Klotz, G. Hamel, W. G. Marshall, R. J. Nelmes, and J. S. Loveday, "Structural instability in ice VIII under pressure," Phys. Rev. Lett. 78, 3141-3144 (1997).

${ }^{58}$ R. J. Nelmes, J. S. Loveday, T. Strässle, C. L. Bull, M. Guthrie, G. Hamel, and S. Klotz, "Annealed high-density amorphous ice under pressure," Nat. Phys. 2, 414-418 (2006).

${ }^{59}$ I. Errea, M. Calandra, and F. Mauri, "Anharmonic free energies and phonon dispersions from the stochastic self-consistent harmonic approximation: Application to platinum and palladium hydrides," Phys. Rev. B 89, 064302 (2014).

${ }^{60}$ R. Bianco, I. Errea, L. Paulatto, M. Calandra, and F. Mauri, "Second-order structural phase transitions, free energy curvature, and temperature-dependent anharmonic phonons in the self-consistent harmonic approximation: Theory and stochastic implementation," Phys. Rev. B 96, 014111 (2017).

${ }^{61}$ L. Monacelli, I. Errea, M. Calandra, and F. Mauri, "Pressure and stress tensor of complex anharmonic crystals within the stochastic self-consistent harmonic approximation," Phys. Rev. B 98, 024106 (2018).

${ }^{62}$ L. Monacelli and F. Mauri, "Time-dependent self-consistent harmonic approximation: Anharmonic nuclear quantum dynamics and time correlation functions," Phys. Rev. B 103, 104305 (2021).

${ }^{63}$ M. Born and R. Oppenheimer, "Zur quantentheorie der molekeln," Ann. Phys. 389, 457-484 (1927).

${ }^{64}$ B. Cheng, E. A. Engel, J. Behler, C. Dellago, and M. Ceriotti, "Ab initio thermodynamics of liquid and solid water," Proc. Natl. Acad. Sci. U. S. A. 116, 1110-1115 (2019).

${ }^{65} \mathrm{Y}$. Zhang and W. Yang, "Comment on 'Generalized gradient approximation made simple,” Phys. Rev. Lett. 80, 890 (1998).

${ }^{66} \mathrm{C}$. Adamo and V. Barone, "Toward reliable density functional methods without adjustable parameters: The PBE0 model," J. Chem. Phys. 110, 6158-6170 (1999).

${ }^{67} \mathrm{~L}$. Goerigk and S. Grimme, "A thorough benchmark of density functional methods for general main group thermochemistry, kinetics, and noncovalent interactions," Phys. Chem. Chem. Phys. 13, 6670 (2011).

${ }^{68}$ S. Grimme, J. Antony, S. Ehrlich, and H. Krieg, "A consistent and accurate $a b$ initio parametrization of density functional dispersion correction (DFT-D) for the 94 elements H-Pu," J. Chem. Phys. 132, 154104 (2010).
${ }^{69}$ V. Kapil, E. Engel, M. Rossi, and M. Ceriotti, "Assessment of approximate methods for anharmonic free energies," J. Chem. Theory Comput. 15, 5845-5857 (2019).

${ }^{70}$ J. Matas, J. Bass, Y. Ricard, E. Mattern, and M. S. T. Bukowinski, "On the bulk composition of the lower mantle: Predictions and limitations from generalized inversion of radial seismic profiles," Geophys. J. Int. 170, 764-780 (2007).

${ }^{71}$ J. C. E. Irving, S. Cottaar, and V. Lekić, "Seismically determined elastic parameters for Earth's outer core," Sci. Adv. 4, eaar2538 (2018).

${ }^{72}$ E. A. Engel, B. Monserrat, and R. J. Needs, "Anharmonic nuclear motion and the relative stability of hexagonal and cubic ice," Phys. Rev. X 5, 021033 (2015).

${ }^{73}$ R. Bianco, I. Errea, M. Calandra, and F. Mauri, "High-pressure phase diagram of hydrogen and deuterium sulfides from first principles: Structural and vibrational properties including quantum and anharmonic effects," Phys. Rev. B 97, 214101 (2018).

${ }^{74}$ J. P. Perdew, K. Burke, and M. Ernzerhof, "Generalized gradient approximation made simple," Phys. Rev. Lett. 77, 3865-3868 (1996).

${ }^{75}$ S. Yoo, X. C. Zeng, and S. S. Xantheas, "On the phase diagram of water with density functional theory potentials: The melting temperature of ice $I_{h}$ with the Perdew-Burke-Ernzerhof and Becke-Lee-Yang-Parr functionals," J. Chem. Phys. 130, 221102 (2009).

${ }^{76}$ A. J. Rusnak, E. R. Pinnick, C. E. Calderon, and F. Wang, "Static dielectric constants and molecular dipole distributions of liquid water and ice-Ih investigated by the PAW-PBE exchange-correlation functional," J. Chem. Phys. 137, 034510 (2012).

${ }^{77}$ L. Monacelli, I. Errea, M. Calandra, and F. Mauri, "Black metal hydrogen above 360 GPa driven by proton quantum fluctuations," Nat. Phys. 17, 63-67 (2020).

${ }^{78} \mathrm{P}$. Vinet, J. R. Smith, J. Ferrante, and J. H. Rose, "Temperature effects on the universal equation of state of solids," Phys. Rev. B 35, 1945-1953 (1987).

${ }^{79}$ P. Giannozzi, S. Baroni, N. Bonini, M. Calandra, R. Car, C. Cavazzoni, D. Ceresoli, G. L. Chiarotti, M. Cococcioni, I. Dabo, A. Dal Corso, S. de Gironcoli, S. Fabris, G. Fratesi, R. Gebauer, U. Gerstmann, C. Gougoussis, A. Kokalj, M. Lazzeri, L. Martin-Samos, N. Marzari, F. Mauri, R. Mazzarello, S. Paolini, A. Pasquarello, L. Paulatto, C. Sbraccia, S. Scandolo, G. Sclauzero, A. P. Seitsonen, A. Smogunov, P. Umari, and R. M. Wentzcovitch, "QUANTUM ESPRESSO: A modular and open-source software project for quantum simulations of materials," J. Phys.: Condens. Matter 21, 395502 (2009).

${ }^{80}$ I. Errea, M. Calandra, C. J. Pickard, J. R. Nelson, R. J. Needs, Y. Li, H. Liu, Y. Zhang, Y. Ma, and F. Mauri, "Quantum hydrogen-bond symmetrization in the superconducting hydrogen sulfide system," Nature 532, 81-84 (2016).

${ }^{81}$ L. Paulatto, I. Errea, M. Calandra, and F. Mauri, "First-principles calculations of phonon frequencies, lifetimes, and spectral functions from weak to strong anharmonicity: The example of palladium hydrides," Phys. Rev. B 91, 054304 (2015).

${ }^{82}$ L. Monacelli, R. Bianco, M. Cherubini, M. Calandra, I. Errea, and F. Mauri, "The stochastic self-consistent harmonic approximation: Calculating vibrational properties of materials with full quantum and anharmonic effects," J. Phys. Condens. Matter 33, 363001 (2021). 